\title{
On the Influence of Solvent Properties on the Structural Characteristics of Molecular Crystal Polymorphs
}

\section{Supporting Information}

\author{
Pavel N. Zolotarev ${ }^{a}$, Nadezhda A. Nekrasova ${ }^{a}$ \\ aSamara Center for Theoretical Materials Science, Samara State Technical University, \\ Molodogvardeyskaya Str. 244, 443100 Samara, Russia
}

\section{Table of contents}

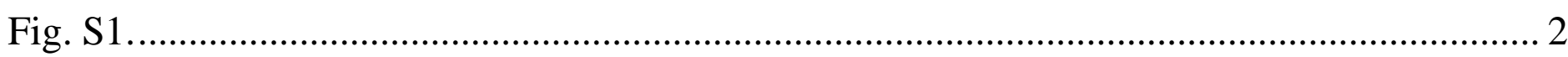

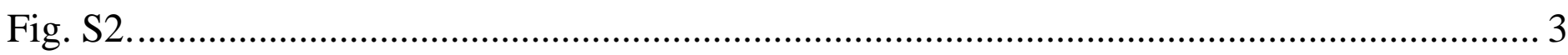

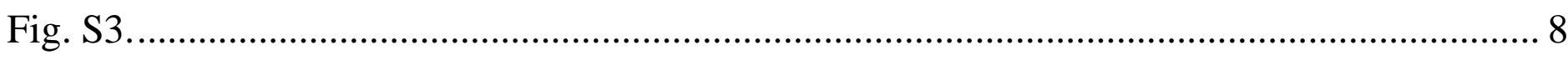

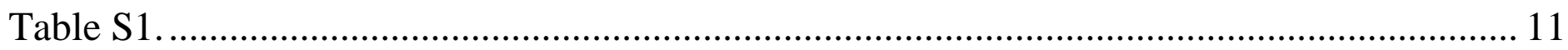

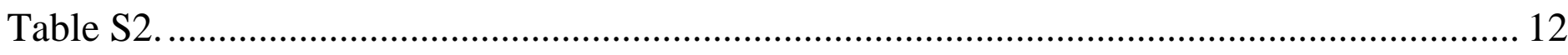

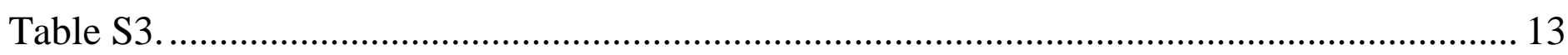

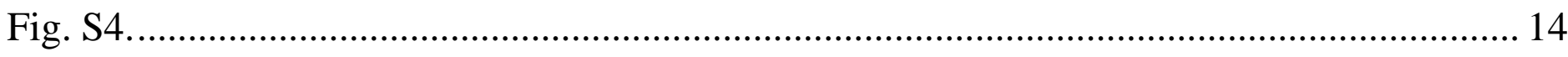

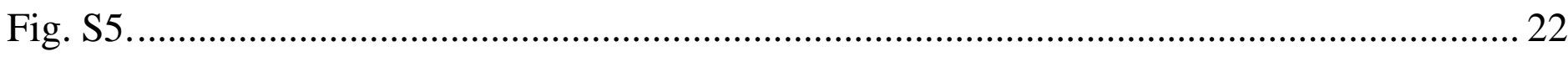

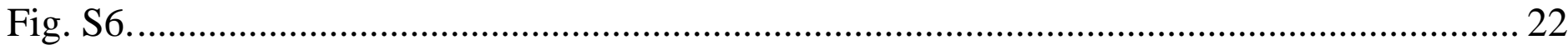


Fig. S1. Heatmap of the distance matrix for solvents in 62-dimensional feature space. 2,2-dimethoxypropane, acetic anhydride and diethyl suberate do not appear because of the absence of multiple descriptor values for these solvents. Euclidean distance was used to evaluate the similarity of solvents in the established feature space. The Euclidean distance values are rounded to integers. Values of the solvent descriptors were rescaled prior to distance calculations as follows: $d_{i}^{\text {std }}=d_{i} / \sigma_{D}$, where $d_{i}^{\text {std }}$ rescaled value of a descriptor D for $i$-th solvent, $d_{i}-$ initial value of a descriptor $\mathrm{D}$ for $i$-th solvent, $\sigma_{D}$ - sample standard deviation of a descriptor $\mathrm{D}$ values.

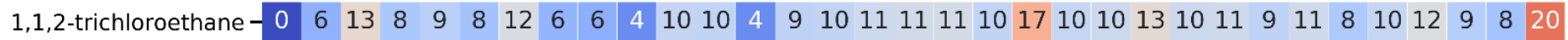

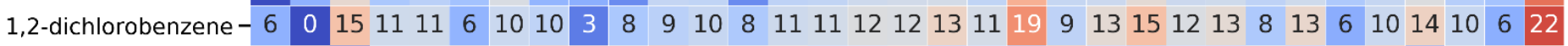

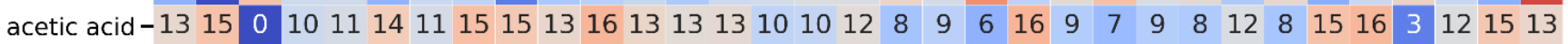

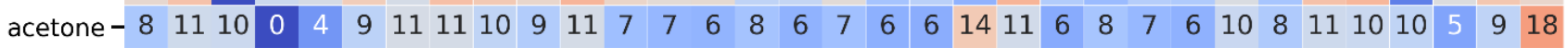
acetonitrile- $9 \begin{array}{lllllllllllllllllllllllllllllllll}11 & 11 & 4 & 0 & 10 & 12 & 12 & 10 & 10 & 12 & 9 & 8 & 8 & 9 & 7 & 7 & 8 & 8 & 15 & 12 & 8 & 9 & 8 & 8 & 11 & 8 & 11 & 11 & 11 & 7 & 10 & 17\end{array}$

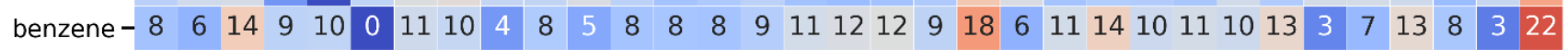

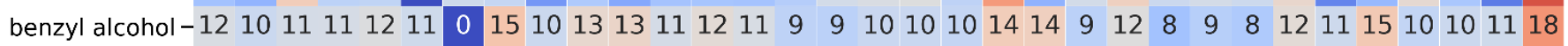

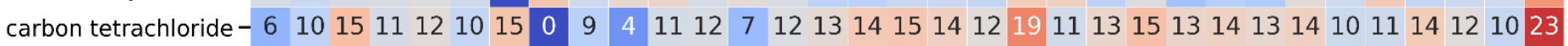

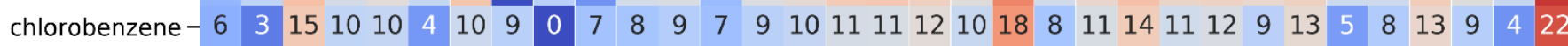

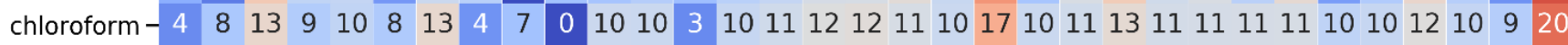

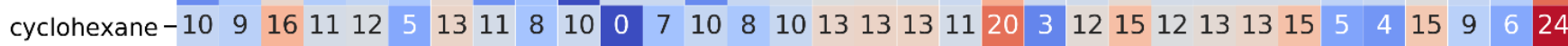

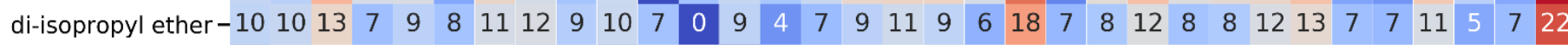

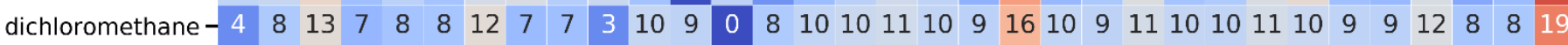

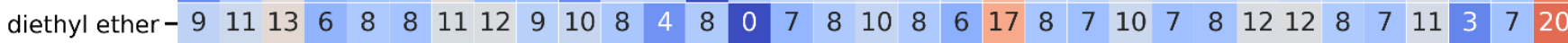

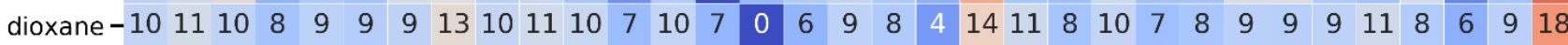

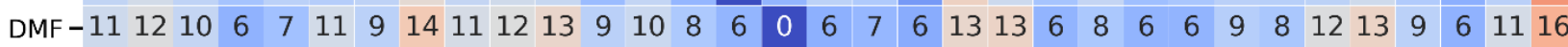

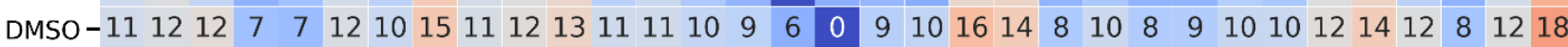

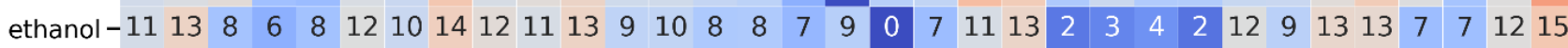

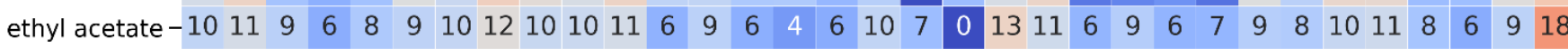
formic acid-17 $19 \quad 6 \quad 141518141918172018161714131611 \quad 13 \quad 0 \quad 201310131215101920 \quad 8 \quad 161911$

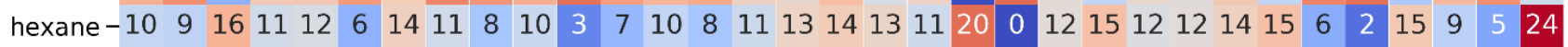

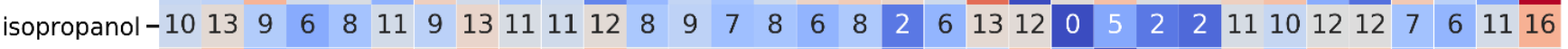

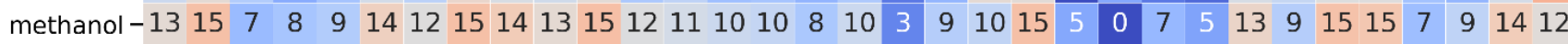

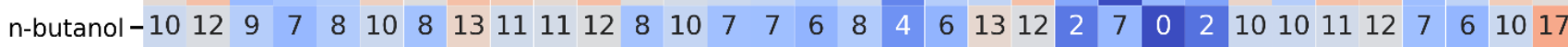

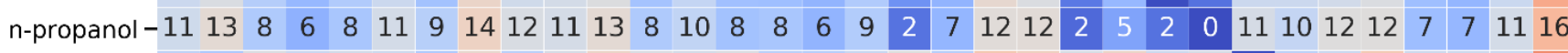

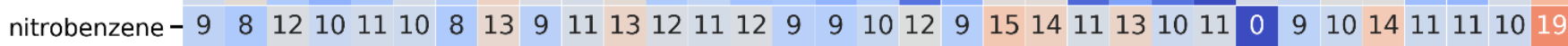

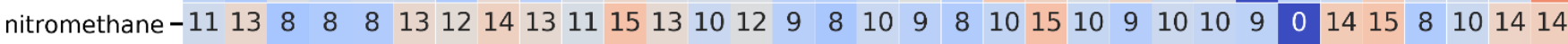

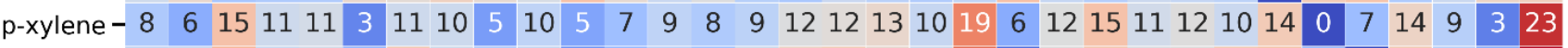

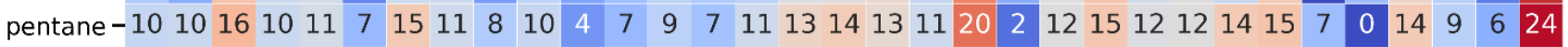

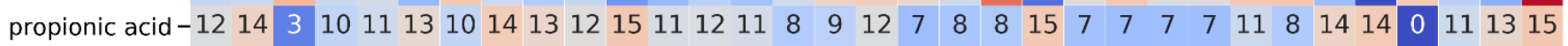

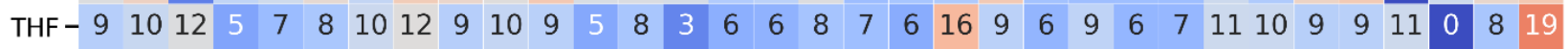

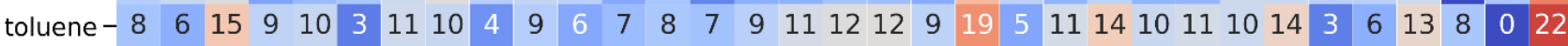
water-20 $22131817221823222024221920181618151811241612171619142324151922 \quad 0$

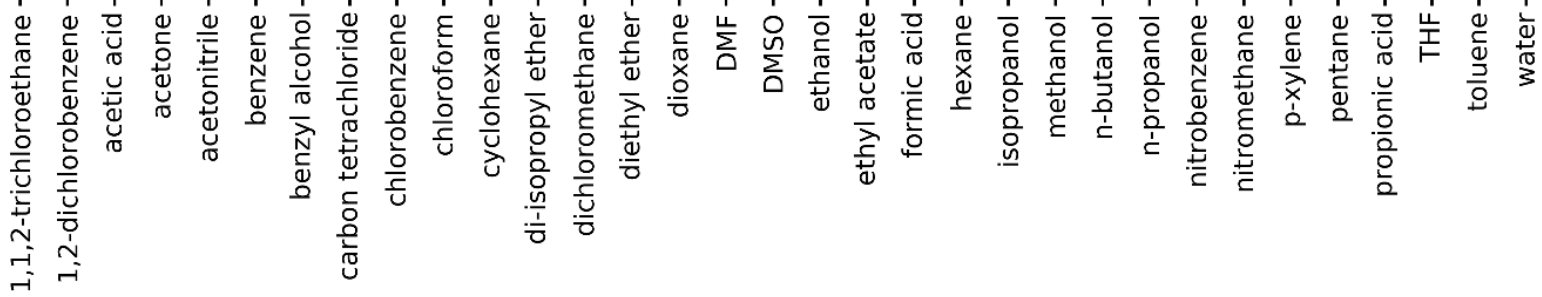


Fig. S2. Histograms and bar-plots of crystal descriptor difference in SSP and DSP subsets. On top of each plot the p-values (prior to Bonferroni correction!) of U test statistic are shown.

- Non-conformational polymorphs (RMSD between crystal molecules in pair of polymorph crystal structures is less than $0.375 \AA$ ).

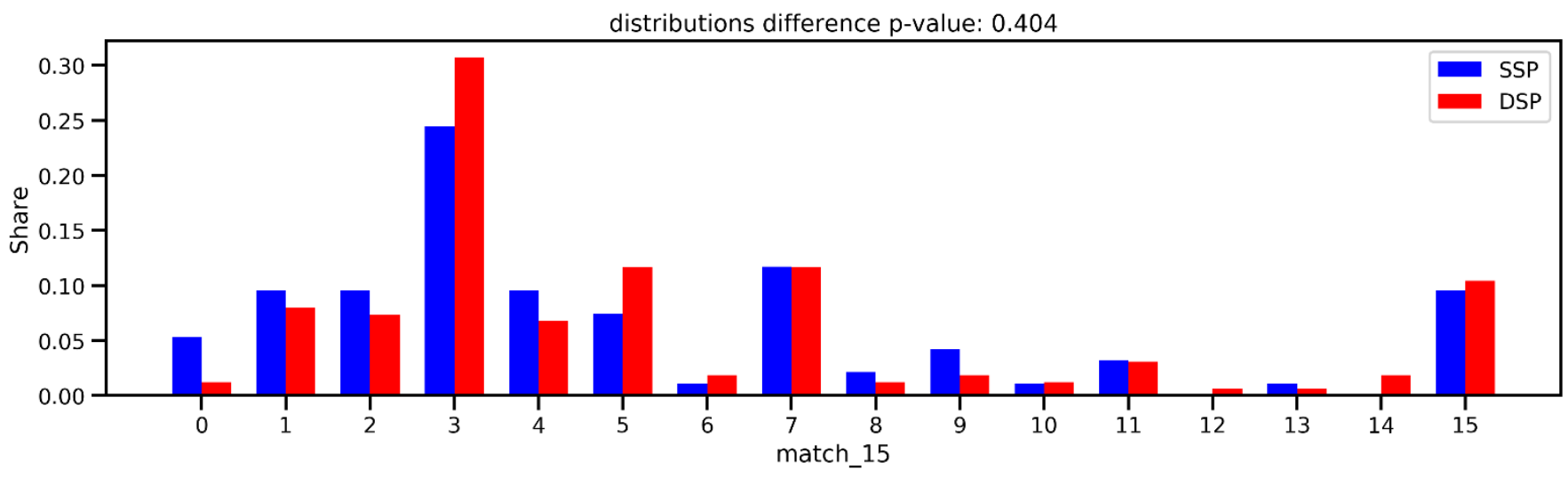

distributions difference p-value: 0.065
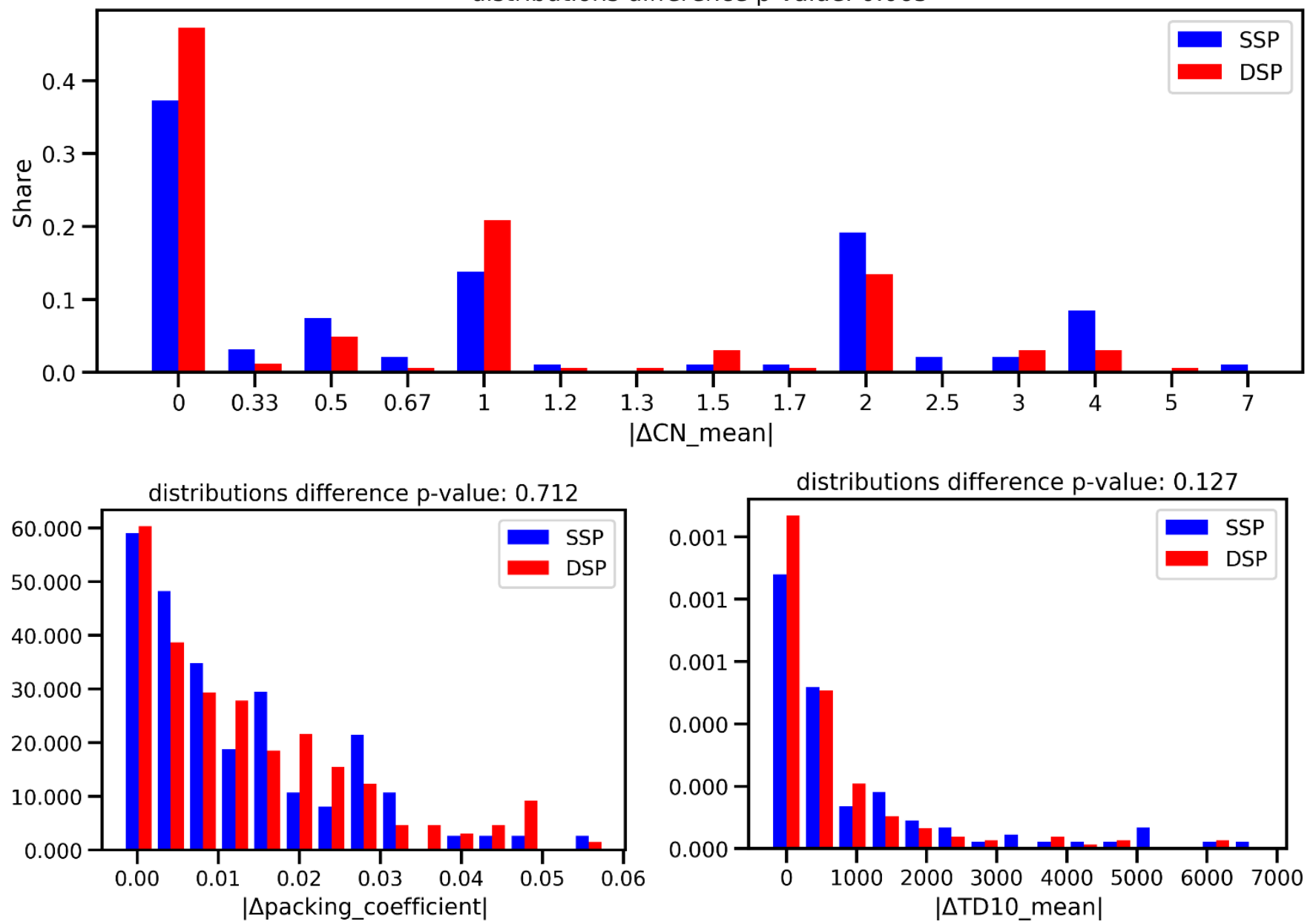

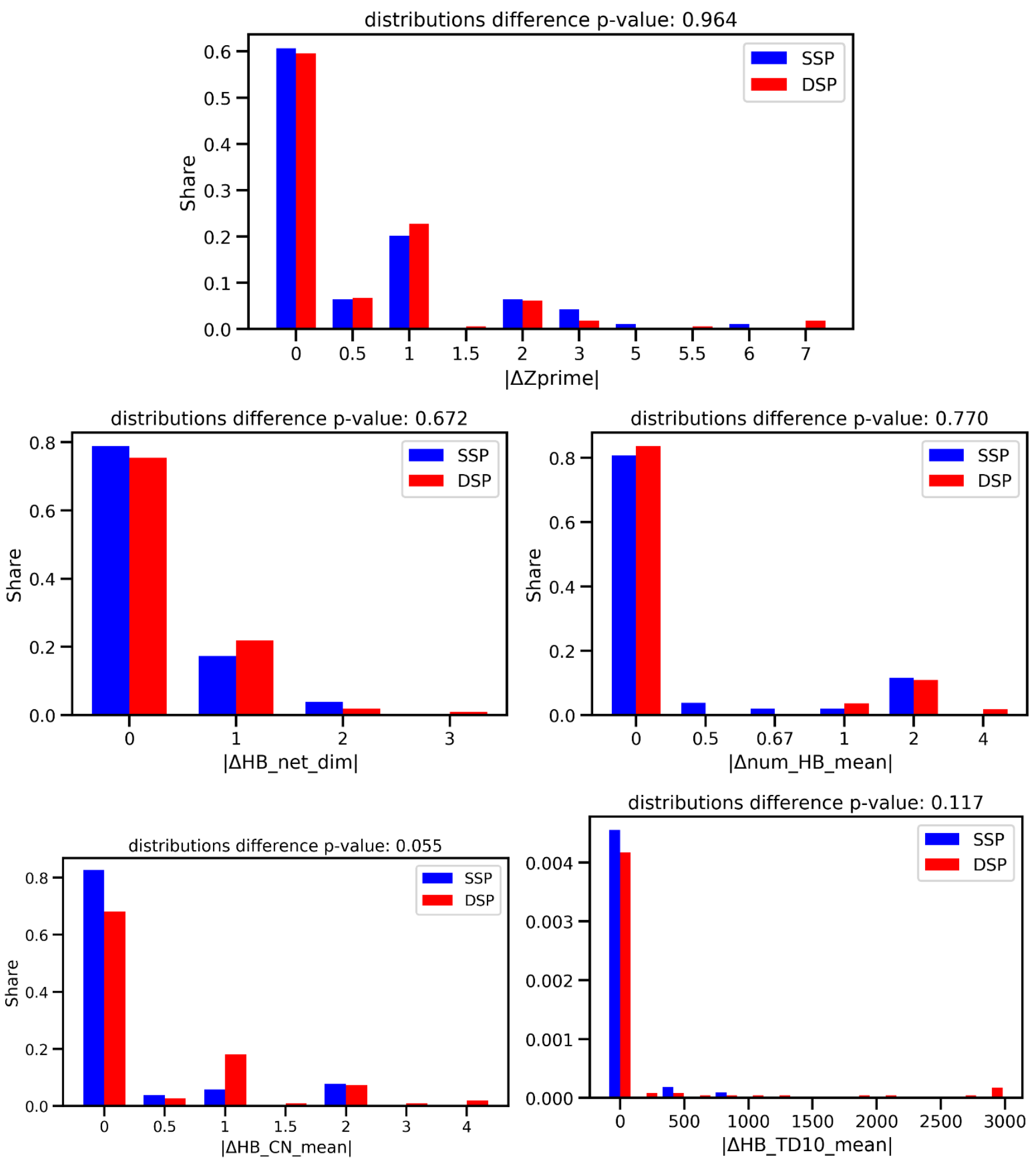

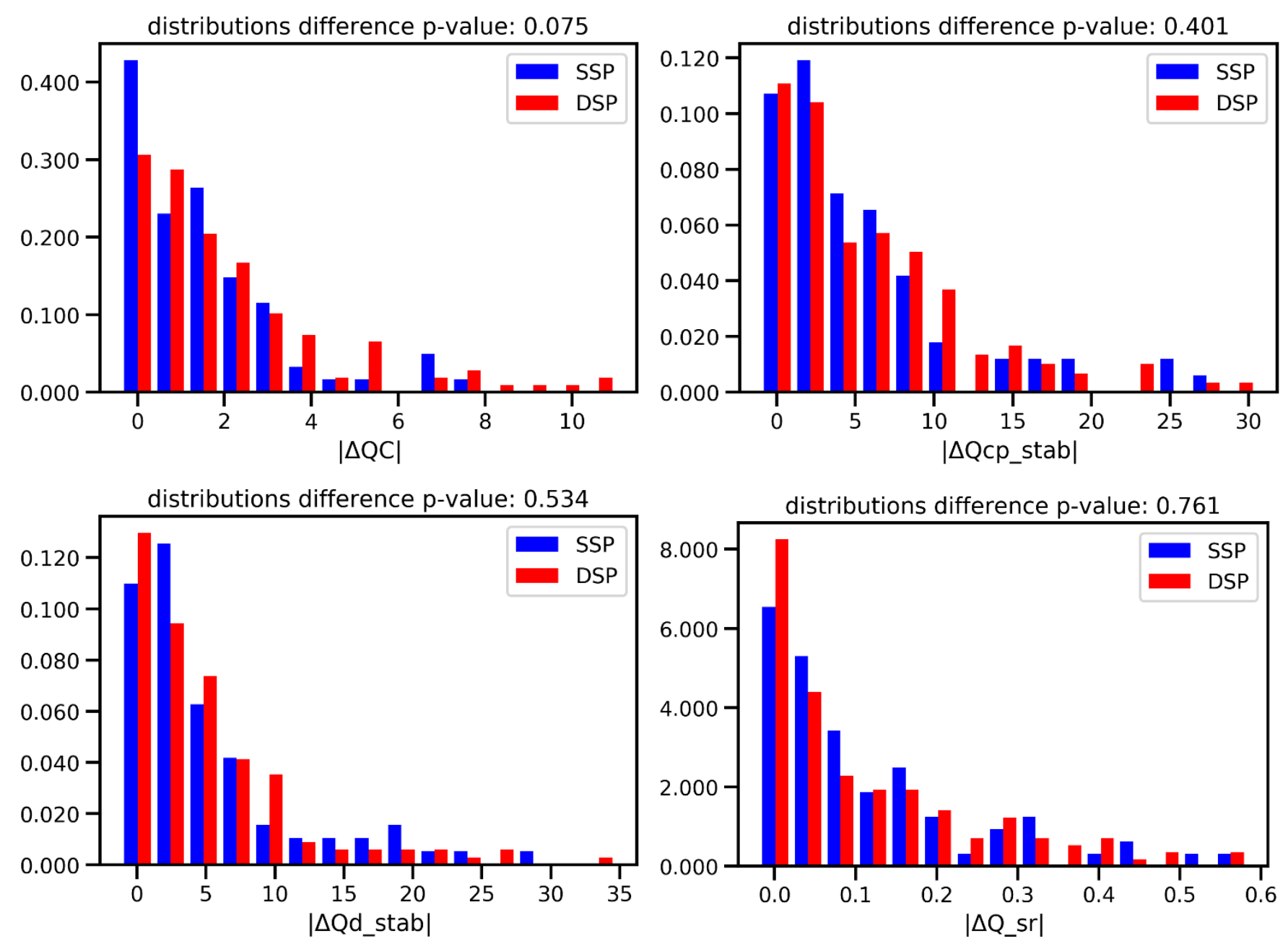

- Conformational polymorphs (RMSD between crystal molecules in pair of polymorph crystal structures is more than $0.375 \AA$ )

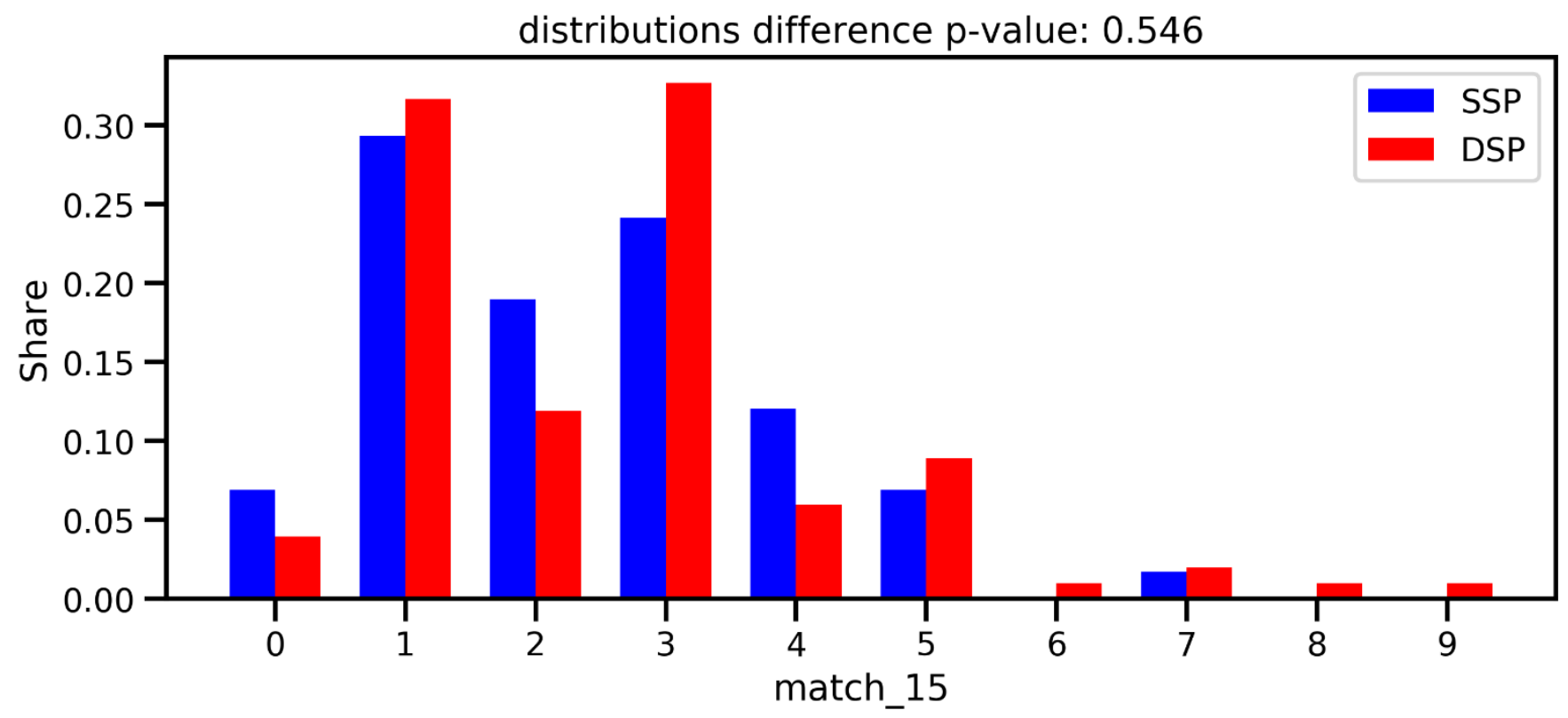



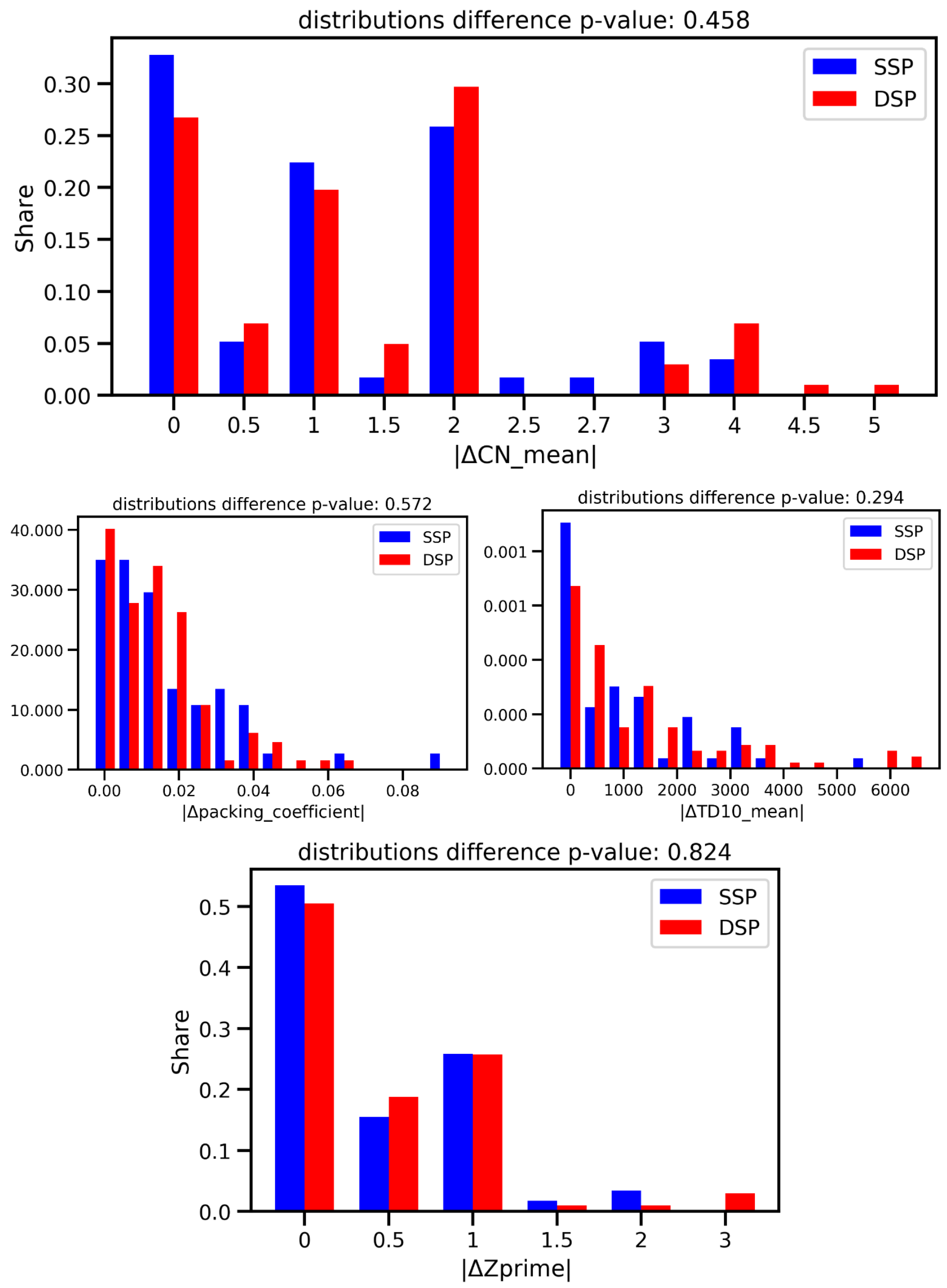

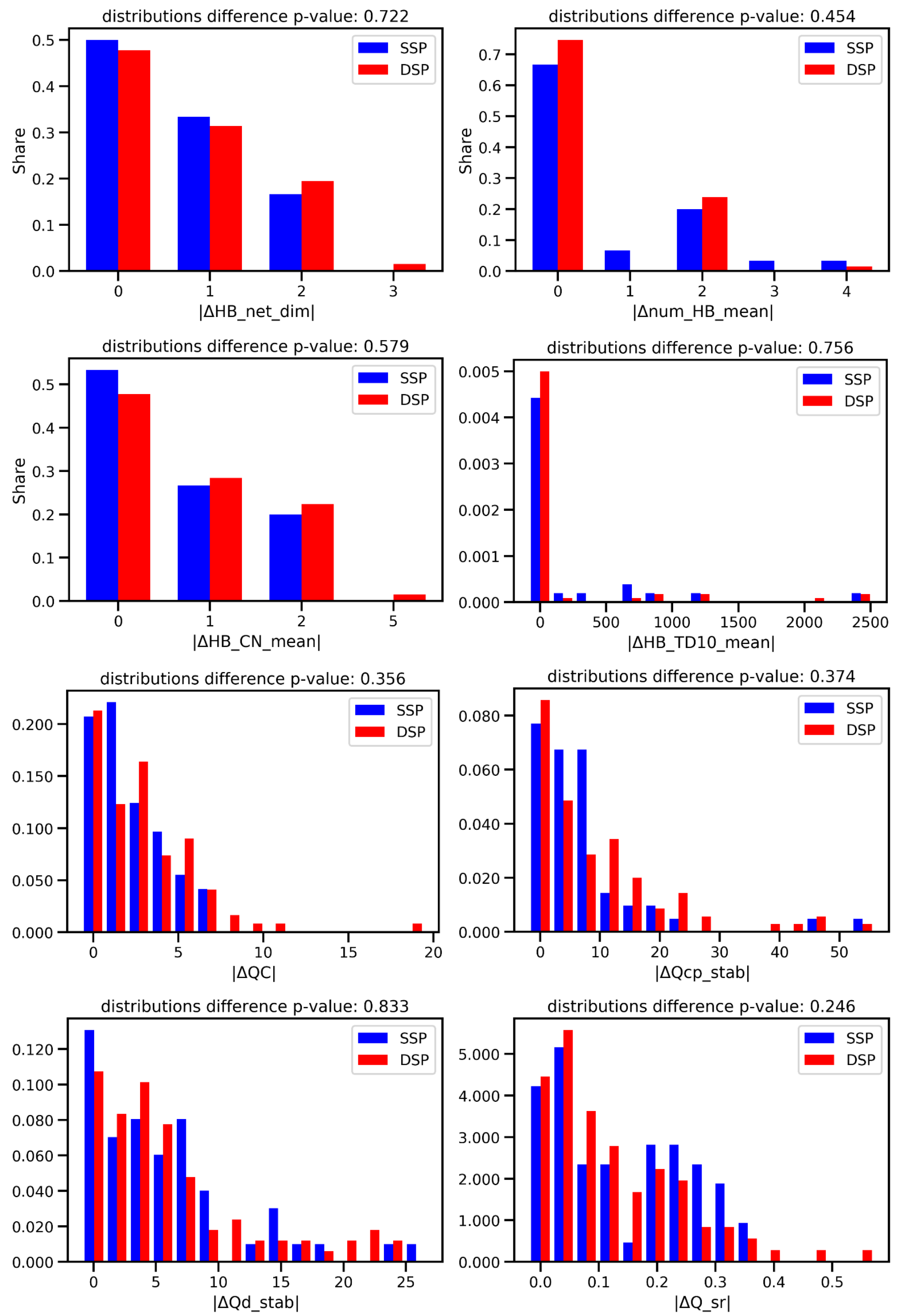
Fig. S3. Combined histograms and kernel density estimates (dashed lines) of descriptors characterizing molecules in SSP (122 molecules - for one molecule of 4-nitroquinoline-1-oxide compound descriptors could not be calculated) and DSP (194 molecules) subsets. Selected descriptors reflect the following molecular properties: atomic types (shares of $\mathrm{C}, \mathrm{N}, \mathrm{O}$ atoms of different hybridization types relative to heavy atom counts), composition (ratio of HB donor and acceptor atoms D/A_ratio, molecular weight MW, number of atoms nAtom), size and topology (Kier's shape indices Kier1/Kier2/Kier3, molecular volume VMcGowan, molecular surface area LabuteASA, share of rotatable bonds RotRatio), polarity (molecular refractivity SMR, partition coefficient SLogP, polar surface area TopoPSA). A vertical solid line of corresponding colour shows a median value of a given descriptor in a given subset. The standard parameters of the kdeplot function (seaborn Python library) were used for kernel density estimates with gaussian kernel. Molecular descriptors were calculated using Mordred library.
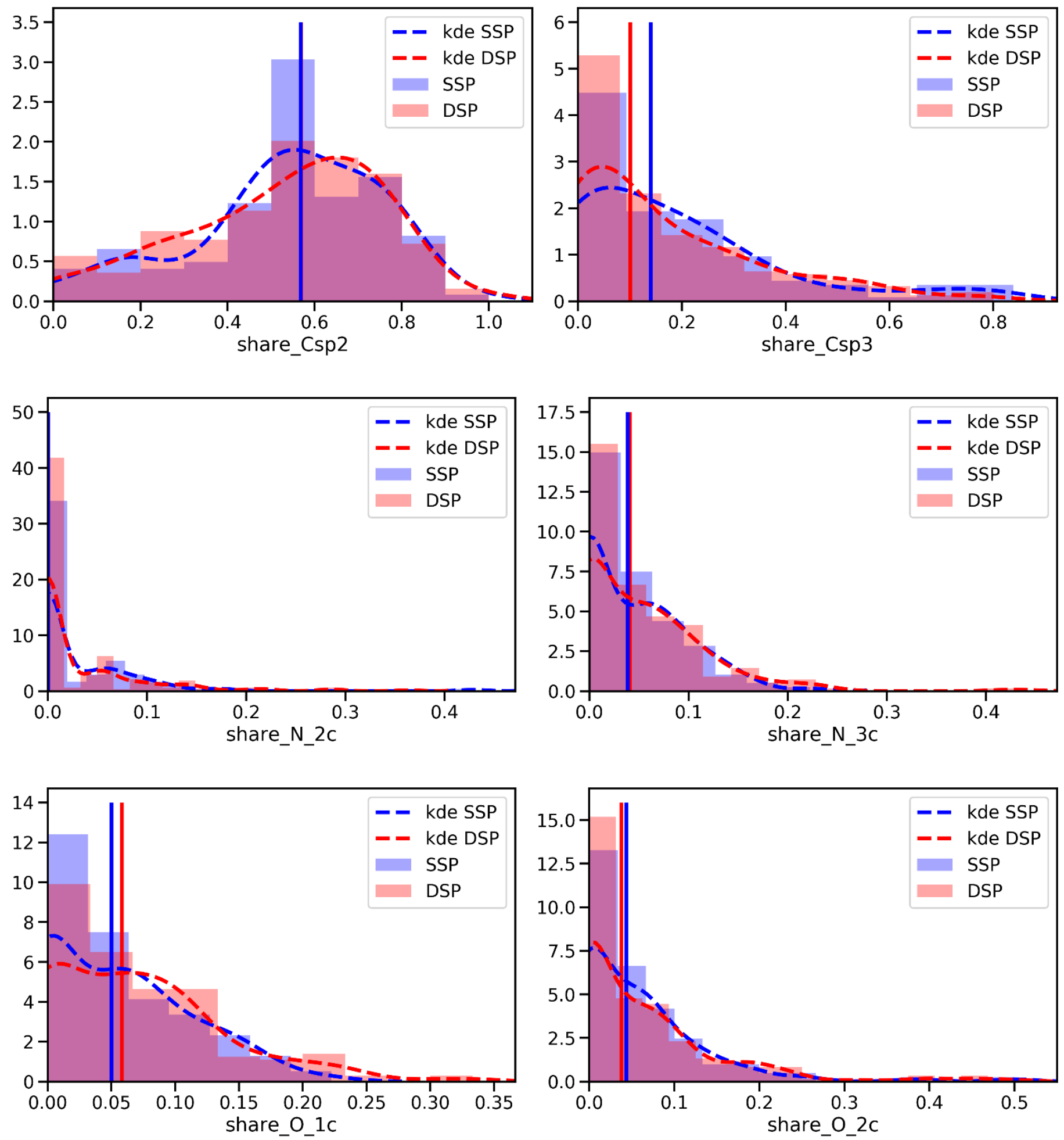

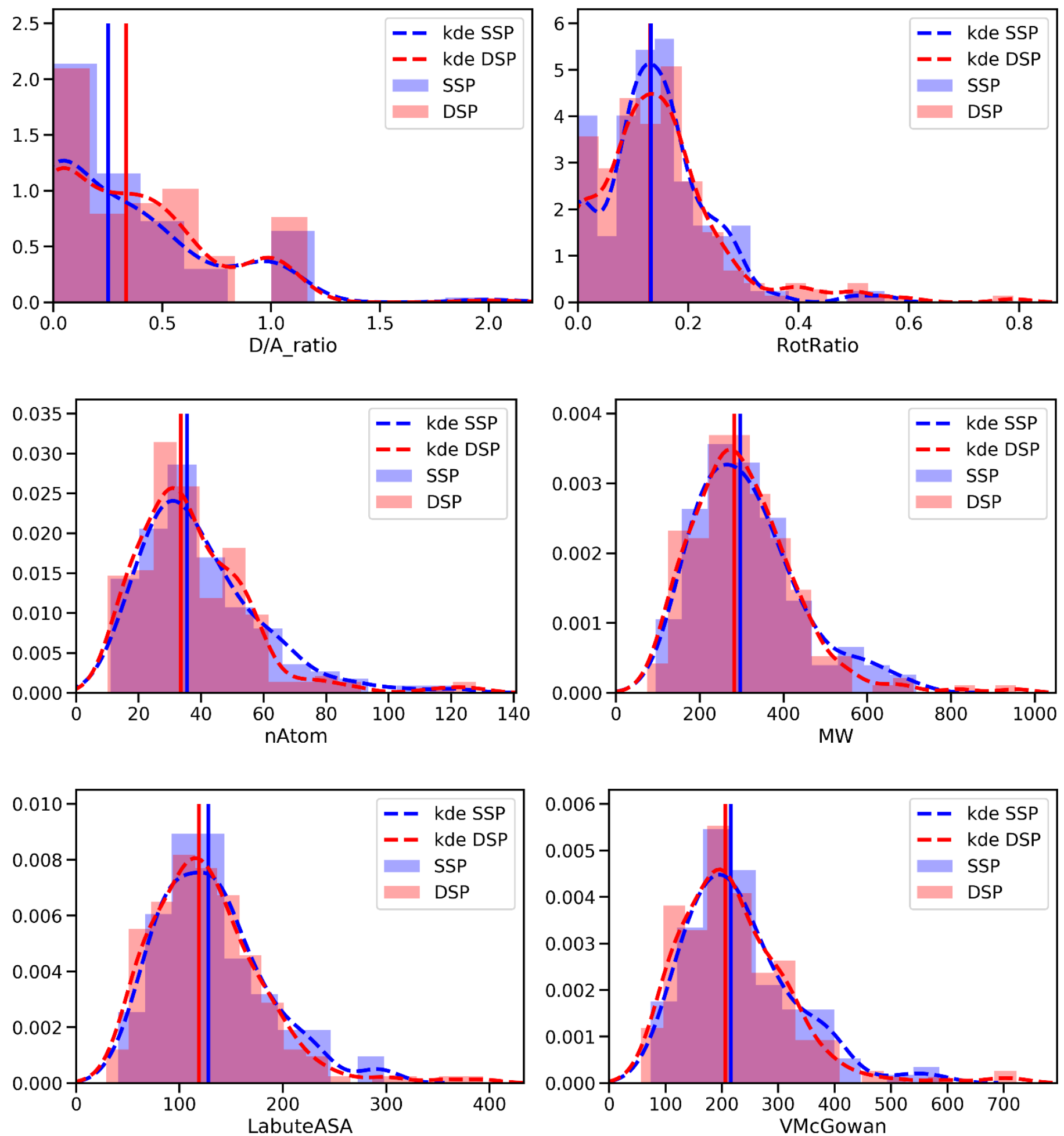

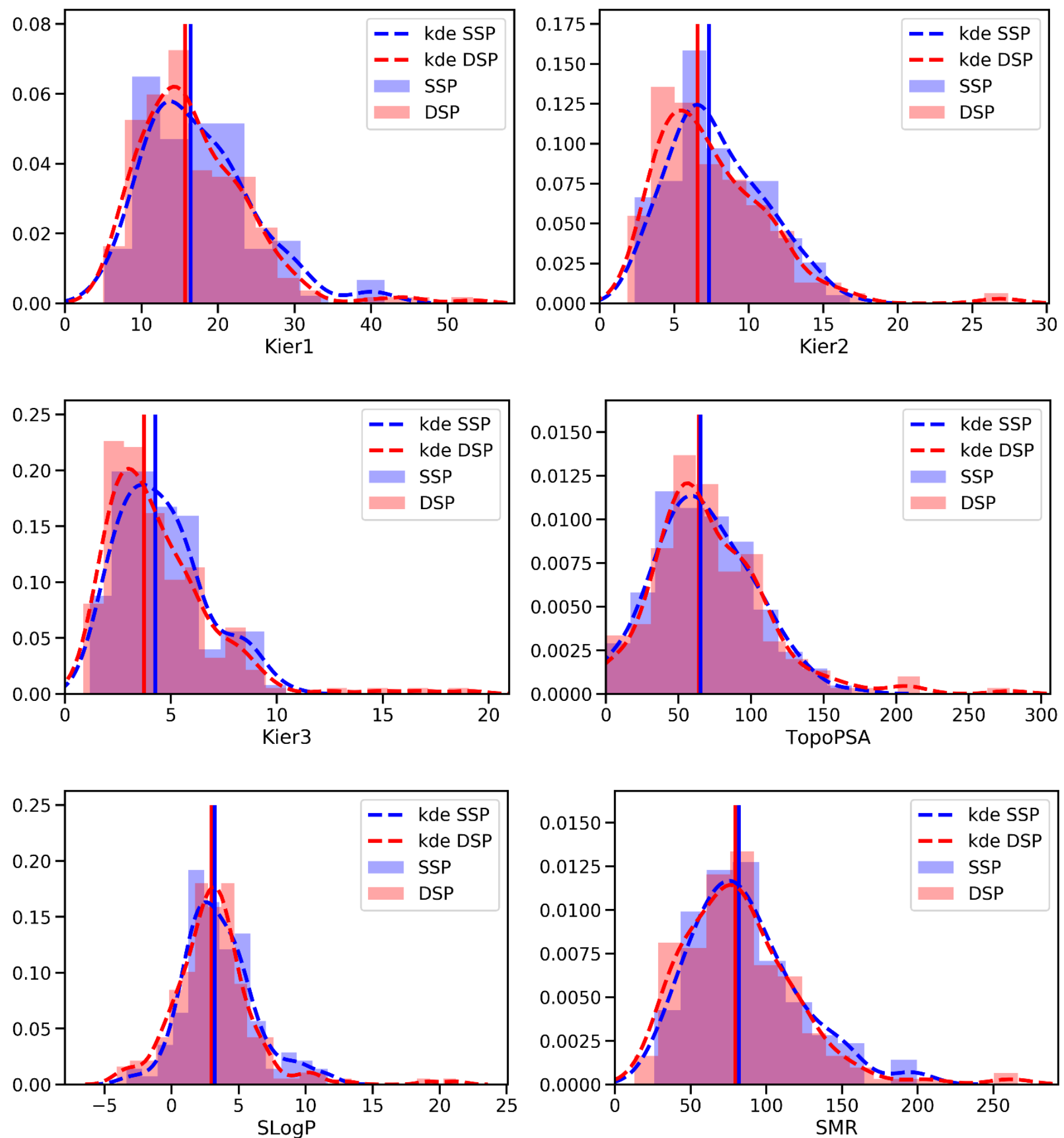
Table S1. Characteristics of crystal descriptor difference distributions in single solvent polymorphs (SSP) and different solvent polymorphs (DSP) subsets. N(SSP) and N(DSP) correspond to the number of polymorph pairs in SSP and DSP subsets, respectively. Mean and median values of DD distributions are presented in corresponding columns. The $p$-values of Mann-Whitney $\mathrm{U}$ test statistic assessing the equality of DD distributions in the two subsets are reported in the $p$-value column. The $p$-value corrected column shows the $\mathrm{p}$-value after the Bonferroni correction procedure.

\begin{tabular}{|c|c|c|c|c|c|c|c|c|}
\hline \multicolumn{9}{|c|}{ Polymorphs (RMSD < $0.375 \AA)$} \\
\hline $\begin{array}{l}\text { descriptor } \\
\text { difference }\end{array}$ & $\mathbf{N}(\mathbf{S S P})$ & $\mathbf{N}(\mathbf{D S P})$ & $\begin{array}{c}\text { mean } \Delta \\
(\text { SSP })\end{array}$ & $\begin{array}{c}\text { mean } \Delta \\
\text { (DSP) }\end{array}$ & $\begin{array}{c}\text { median } \Delta \\
(\text { SSP) }\end{array}$ & $\begin{array}{c}\text { median } \Delta \\
\text { (DSP) }\end{array}$ & $p$-value & $\begin{array}{c}p \text {-value } \\
\text { corrected }\end{array}$ \\
\hline$\Delta$ Zprime & 94 & 163 & 0.6064 & 0.6104 & 0 & 0 & 0.964 & 1.0 \\
\hline$\Delta$ num_HB_mean & 52 & 110 & 0.2821 & 0.3273 & 0 & 0 & 0.770 & 1.0 \\
\hline$\Delta \mathrm{Q} \_\mathrm{sr}$ & 80 & 142 & 0.1344 & 0.1349 & 0.0866 & 0.0801 & 0.761 & 1.0 \\
\hline$\Delta$ packing_coefficient & 94 & 163 & 0.0142 & 0.0152 & 0.0106 & 0.0116 & 0.712 & 1.0 \\
\hline$\Delta \mathrm{HB} \_$net_dim & 52 & 110 & 0.2500 & 0.2818 & 0 & 0 & 0.672 & 1.0 \\
\hline$\Delta \mathrm{Qd} \_s t a b$ & 80 & 142 & 6.5035 & 6.0892 & 4.3429 & 4.1493 & 0.534 & 1.0 \\
\hline match_15 & 94 & 163 & 5.2447 & 5.6258 & 4 & 4 & 0.404 & 1.0 \\
\hline$\Delta$ Qcp_stab & 80 & 142 & 6.3897 & 6.9002 & 4.4225 & 5.0727 & 0.401 & 1.0 \\
\hline$\Delta$ TD10_mean & 94 & 163 & 1176.7 & 817.4 & 629 & 445 & 0.127 & 1.0 \\
\hline$\Delta$ HB_TD10_mean & 52 & 110 & 52.0 & 250.7 & 0 & 0 & 0.117 & 1.0 \\
\hline$\Delta \mathrm{QC}$ & 80 & 142 & 1.8593 & 2.4168 & 1.4949 & 1.7350 & 0.075 & 0.978 \\
\hline$\Delta$ CN_mean & 94 & 163 & 1.1622 & 0.8287 & 0.8333 & 0.5000 & 0.065 & 0.842 \\
\hline$\Delta \mathrm{HB} \_\mathrm{CN} \_$mean & 52 & 110 & 0.2308 & 0.4545 & 0 & 0 & 0.055 & 0.714 \\
\hline \multicolumn{9}{|c|}{ Conformational polymorphs (RMSD $>0.375 \AA)$} \\
\hline $\begin{array}{l}\text { descriptor } \\
\text { difference }\end{array}$ & $\mathbf{N}(\mathbf{S S P})$ & $\mathbf{N}(\mathbf{D S P})$ & $\begin{array}{c}\text { mean } \Delta \\
(\mathrm{SSP})\end{array}$ & $\begin{array}{c}\text { mean } \Delta \\
(\text { DSP })\end{array}$ & $\begin{array}{c}\text { median } \Delta \\
(\text { SSP) }\end{array}$ & $\begin{array}{c}\text { median } \Delta \\
(\text { DSP) }\end{array}$ & $p$-value & $\begin{array}{c}p \text {-value } \\
\text { corrected }\end{array}$ \\
\hline$\Delta \mathrm{Qd} \_s t a b$ & 54 & 91 & 6.7009 & 6.9634 & 5.2951 & 5.2788 & 0.833 & 1.0 \\
\hline$\Delta$ Zprime & 58 & 101 & 0.4310 & 0.4752 & 0 & 0 & 0.824 & 1.0 \\
\hline$\Delta$ HB_TD10_mean & 30 & 67 & 240.4 & 207.5 & 2 & 19 & 0.756 & 1.0 \\
\hline$\Delta$ HB_net_dim & 30 & 67 & 0.6667 & 0.7463 & 0.5 & 1 & 0.722 & 1.0 \\
\hline$\Delta$ HB_CN_mean & 30 & 67 & 0.6667 & 0.8060 & 0 & 1 & 0.579 & 1.0 \\
\hline$\Delta$ packing_coefficient & 58 & 101 & 0.0196 & 0.0171 & 0.0156 & 0.0153 & 0.572 & 1.0 \\
\hline match_15 & 58 & 101 & 2.3448 & 2.5842 & 2 & 3 & 0.546 & 1.0 \\
\hline$\Delta \mathrm{CN} \_$mean & 58 & 101 & 1.1753 & 1.3614 & 1 & 1 & 0.458 & 1.0 \\
\hline$\Delta$ num_HB_mean & 30 & 67 & 0.7000 & 0.5373 & 0 & 0 & 0.454 & 1.0 \\
\hline$\Delta$ Qcp_stab & 54 & 91 & 9.0716 & 11.1862 & 7.1641 & 7.4626 & 0.374 & 1.0 \\
\hline$\Delta \mathrm{QC}$ & 54 & 91 & 2.8201 & 3.4819 & 2.3090 & 3.1163 & 0.356 & 1.0 \\
\hline$\Delta$ TD10_mean & 58 & 101 & 1179.6 & 1486.8 & 844 & 910 & 0.294 & 1.0 \\
\hline$\Delta \mathrm{Q} \_s r$ & 54 & 91 & 0.1612 & 0.1412 & 0.1380 & 0.1058 & 0.246 & 1.0 \\
\hline
\end{tabular}


Table S2. Characteristics of crystal descriptor difference distributions in 21 compounds common to single solvent polymorphs (SSP) and different solvent polymorphs (DSP) subsets. N(SSP) and N(DSP) correspond to the number of polymorph pairs in SSP and DSP subsets, respectively. Mean and median values of DD distributions are given in corresponding columns. The $p$-values of Mann-Whitney $\mathrm{U}$ test statistic assessing the equality of DD distributions in two subsets are reported in the $p$-value column. The $p$-value corrected column shows the $\mathrm{p}$-value after the Bonferroni correction procedure.

\begin{tabular}{|c|c|c|c|c|c|c|c|c|}
\hline \multicolumn{9}{|c|}{ Polymorphs $($ RMSD $<0.375 \AA)$} \\
\hline $\begin{array}{l}\text { descriptor } \\
\text { difference }\end{array}$ & $\mathbf{N}(\mathbf{S S P})$ & $\mathbf{N}(\mathbf{D S P})$ & $\begin{array}{c}\operatorname{mean} \Delta \\
(\text { SSP })\end{array}$ & $\begin{array}{c}\text { mean } \Delta \\
(\text { DSP })\end{array}$ & $\underset{(\text { SSP })}{\operatorname{median} \Delta}$ & $\begin{array}{c}\operatorname{median} \Delta \\
\quad(\mathrm{DSP})\end{array}$ & $p$-value & $\begin{array}{c}p \text {-value } \\
\text { corrected }\end{array}$ \\
\hline$\Delta$ packing_coefficient & 18 & 41 & 0.0157 & 0.0163 & 0.0104 & 0.0105 & 0.921 & 1.0 \\
\hline$\Delta$ Qcp_stab & 15 & 35 & 7.6012 & 7.1405 & 3.7358 & 3.7743 & 0.882 & 1.0 \\
\hline$\Delta$ HB_net_dim & 18 & 41 & 0.1667 & 0.1951 & 0 & 0 & 0.807 & 1.0 \\
\hline$\Delta$ Zprime & 18 & 41 & 0.6667 & 0.5488 & 0.25 & 0 & 0.670 & 1.0 \\
\hline$\Delta \mathrm{HB} \_\mathrm{CN} \_$mean & 18 & 41 & 0.2222 & 0.2927 & 0 & 0 & 0.656 & 1.0 \\
\hline$\Delta \mathrm{Q} \_s r$ & 15 & 35 & 0.1049 & 0.1382 & 0.0579 & 0.0677 & 0.641 & 1.0 \\
\hline$\Delta$ num_HB_mean & 18 & 41 & 0.1111 & 0.1951 & 0 & 0 & 0.609 & 1.0 \\
\hline$\Delta \mathrm{QC}$ & 15 & 35 & 2.2819 & 2.6194 & 1.1905 & 1.6987 & 0.582 & 1.0 \\
\hline$\Delta \mathrm{Qd} \_s t a b$ & 15 & 35 & 6.6296 & 6.5662 & 6.3002 & 4.0474 & 0.525 & 1.0 \\
\hline$\Delta \mathrm{HB}$-TD10_mean & 18 & 41 & 15.1667 & 313.2033 & 0 & 0 & 0.434 & 1.0 \\
\hline match_15 & 18 & 41 & 4.2222 & 5.1707 & 3 & 3 & 0.388 & 1.0 \\
\hline$\Delta \mathrm{CN} \_$mean & 18 & 41 & 1.0787 & 0.7581 & 0.8333 & 0.5000 & 0.241 & 1.0 \\
\hline$\Delta$ TD10_mean & 18 & 41 & 925.9769 & 617.1687 & 529.25 & 440 & 0.190 & 1.0 \\
\hline \multicolumn{9}{|c|}{ Conformational polymorphs $($ RMSD $>0.375 \AA)$} \\
\hline $\begin{array}{l}\text { descriptor } \\
\text { difference }\end{array}$ & $\mathbf{N}($ SSP $)$ & $\mathbf{N}(\mathbf{D S P})$ & $\begin{array}{c}\operatorname{mean} \Delta \\
(\mathrm{SSP})\end{array}$ & $\begin{array}{c}\text { mean } \Delta \\
(\mathrm{DSP})\end{array}$ & $\underset{(\text { SSP })}{\operatorname{median} \Delta}$ & $\underset{(\text { DSP })}{\operatorname{median}} \Delta$ & $p$-value & $\begin{array}{c}p \text {-value } \\
\text { corrected }\end{array}$ \\
\hline$\Delta \mathrm{Qd} \_s t a b$ & 10 & 20 & 6.1635 & 5.7630 & 4.2433 & 4.2461 & 0.947 & 1.0 \\
\hline$\Delta Q \mathrm{Qcp} \_$stab & 10 & 20 & 12.9323 & 13.1147 & 10.1165 & 10.1165 & 0.947 & 1.0 \\
\hline match_15 & 11 & 26 & 2.9091 & 3.0000 & 3 & 3 & 0.852 & 1.0 \\
\hline$\Delta \mathrm{HB} \_C N$ _mean & 10 & 23 & 1.0000 & 1.0870 & 1 & 1 & 0.819 & 1.0 \\
\hline$\Delta$ HB_net_dim & 11 & 26 & 0.8182 & 0.8846 & 1 & 1 & 0.818 & 1.0 \\
\hline$\Delta \mathrm{CN} \_$mean & 11 & 26 & 1.0455 & 1.2885 & 1 & 1 & 0.758 & 1.0 \\
\hline$\Delta$ HB_TD10_mean & 10 & 23 & 398.5000 & 310.3478 & 2 & 19 & 0.588 & 1.0 \\
\hline$\Delta$ num_HB_mean & 10 & 23 & 1.0000 & 0.7826 & 1 & 0 & 0.583 & 1.0 \\
\hline$\Delta$ TD10_mean & 11 & 26 & 806.4091 & 1233.8654 & 656 & 980.25 & 0.495 & 1.0 \\
\hline$\Delta \mathrm{Q} \_s r$ & 10 & 20 & 0.1850 & 0.1490 & 0.1468 & 0.1064 & 0.468 & 1.0 \\
\hline$\Delta$ Zprime & 11 & 26 & 0.3636 & 0.6538 & 0 & 0.5 & 0.446 & 1.0 \\
\hline$\Delta \mathrm{QC}$ & 10 & 20 & 4.2784 & 3.7472 & 4.4731 & 3.5959 & 0.441 & 1.0 \\
\hline$\Delta$ packing_coefficient & 11 & 26 & 0.0191 & 0.0139 & 0.0106 & 0.0098 & 0.335 & 1.0 \\
\hline
\end{tabular}


Table S3. Pairs of crystal and solvent descriptor difference pairs, showing the lowest $p$-values of correlation coefficients in a set of non-conformational polymorphs (crystal molecules RMSD $<0.375 \AA$ ). Column N reports the number polymorph pairs tested for correlation. The $p$-value showing that an observed value of correlation coefficient is significantly different from zero is reported in the $p$-value column. The $p$-value corrected column shows the $p$-value after the Bonferroni correction procedure.

\begin{tabular}{|c|c|c|c|c|c|}
\hline$\Delta$ crystal descriptor & $\Delta$ solvent descriptor & $\mathbf{N}$ & Pearson's $\mathbf{r}$ & $p$-value & $p$-value corrected \\
\hline$\Delta$ packing_coefficient & $\Delta$ dipole moment, $\mathrm{D}$ & 189 & 0.318 & $8.47 \mathrm{E}-06$ & 0.006 \\
\hline$\Delta$ packing_coefficient & $\Delta$ TopoPSA & 190 & 0.272 & $1.44 \mathrm{E}-04$ & 0.107 \\
\hline$\Delta \mathrm{CN} \_m e a n$ & $\Delta$ TopoPSA & 190 & 0.265 & 2.23E-04 & 0.166 \\
\hline$\Delta \mathrm{CN} \_m e a n$ & $\Delta$ dipole moment, $\mathrm{D}$ & 189 & 0.260 & 3.03E-04 & 0.226 \\
\hline$\Delta \mathrm{QC}$ & $\Delta$ TopoPSA & 160 & -0.277 & 4.01E-04 & 0.298 \\
\hline$\Delta$ packing_coefficient & $\triangle \mathrm{nHBAcc}$ & 190 & 0.251 & 4.76E-04 & 0.354 \\
\hline$\Delta$ Zprime & $\Delta$ alpha_Abraham & 188 & 0.251 & $5.08 \mathrm{E}-04$ & 0.378 \\
\hline$\Delta \mathrm{HB} \_n e t \_d i m$ & $\Delta$ ETA_dEpsilon_A & 138 & -0.288 & $6.06 \mathrm{E}-04$ & 0.451 \\
\hline$\Delta$ TD10_mean & $\Delta$ TopoPSA & 190 & 0.241 & 7.95E-04 & 0.591 \\
\hline$\Delta \mathrm{CN} \_$mean & $\triangle \mathrm{nHBAcc}$ & 190 & 0.235 & $1.13 \mathrm{E}-03$ & 0.838 \\
\hline$\Delta$ num_HB_mean & $\Delta$ Freezing point, oC & 138 & 0.273 & $1.19 \mathrm{E}-03$ & 0.889 \\
\hline$\Delta \mathrm{HB} \_n e t \_d i m$ & $\Delta$ FNSA1 & 138 & -0.272 & $1.27 \mathrm{E}-03$ & 0.942 \\
\hline$\Delta$ Zprime & $\Delta$ alpha_KT & 189 & 0.226 & $1.75 \mathrm{E}-03$ & 1.0 \\
\hline$\Delta$ Zprime & $\Delta$ beta_KT & 189 & 0.225 & $1.85 \mathrm{E}-03$ & 1.0 \\
\hline$\Delta$ num_HB_mean & $\Delta$ Basicity SB & 137 & -0.263 & $1.94 \mathrm{E}-03$ & 1.0 \\
\hline$\Delta$ crystal descriptor & $\Delta$ solvent descriptor & $\mathbf{N}$ & Spearman's $\rho$ & $p$-value & $p$-value corrected \\
\hline$\Delta \mathrm{CN} \_m e a n$ & $\Delta$ TopoPSA & 190 & 0.334 & $2.5 \mathrm{E}-06$ & 0.002 \\
\hline$\Delta \mathrm{CN} \_$mean & $\Delta$ dipole moment, $\mathrm{D}$ & 189 & 0.307 & $1.7 \mathrm{E}-05$ & 0.013 \\
\hline$\Delta$ packing_coefficient & $\triangle \mathrm{nHBAcc}$ & 190 & 0.304 & $2.0 \mathrm{E}-05$ & 0.015 \\
\hline$\Delta$ TD10_mean & $\Delta$ TopoPSA & 190 & 0.293 & $4.0 \mathrm{E}-05$ & 0.030 \\
\hline$\Delta$ packing_coefficient & $\Delta$ dipole moment, $\mathrm{D}$ & 189 & 0.289 & $5.4 \mathrm{E}-05$ & 0.041 \\
\hline$\Delta \mathrm{CN} \_$mean & $\triangle \mathrm{nHBAcc}$ & 190 & 0.288 & $5.6 \mathrm{E}-05$ & 0.042 \\
\hline$\Delta$ packing_coefficient & $\Delta$ TopoPSA & 190 & 0.285 & $6.9 \mathrm{E}-05$ & 0.051 \\
\hline$\Delta \mathrm{QC}$ & $\Delta$ TopoPSA & 160 & -0.305 & 8.7E-05 & 0.064 \\
\hline$\Delta H B \_n e t \_d i m$ & $\Delta$ FNSA1 & 138 & -0.318 & $1.4 \mathrm{E}-04$ & 0.106 \\
\hline$\Delta Q c p \_s t a b$ & $\Delta \mathrm{HSP}_{-} \mathrm{p}$ & 158 & -0.278 & 4.0E-04 & 0.296 \\
\hline$\Delta$ Qcp_stab & $\Delta$ TopoPSA & 160 & -0.267 & $6.5 \mathrm{E}-04$ & 0.486 \\
\hline$\Delta \mathrm{QC}$ & $\Delta$ dipole moment, $\mathrm{D}$ & 159 & -0.264 & 7.6E-04 & 0.563 \\
\hline$\Delta$ HB_TD10_mean & $\Delta$ FNSA1 & 138 & -0.280 & 8.7E-04 & 0.645 \\
\hline$\Delta \mathrm{QC}$ & $\Delta \mathrm{HSP}_{\mathrm{N}} \mathrm{p}$ & 158 & -0.260 & $9.6 \mathrm{E}-04$ & 0.714 \\
\hline$\Delta H B \_n e t \_d i m$ & $\Delta$ ETA_dEpsilon_A & 138 & -0.273 & $1.2 \mathrm{E}-03$ & 0.897 \\
\hline
\end{tabular}


Fig. S4. For each pair of crystal and solvent descriptor difference, number of polymorph pairs (plot on the left) and magnitude of Spearman's correlation coefficients (scatterplot on the right) are demonstrated, depending on a maximum RMSD between crystal molecules in the polymorph pair. Additionally, the crosses corresponding to correlation coefficient values are coloured depending on their $p$-value in logarithmic scale. Below separate scatterplots are presented illustrating correlation in DD pair at a given maximum RMSD.
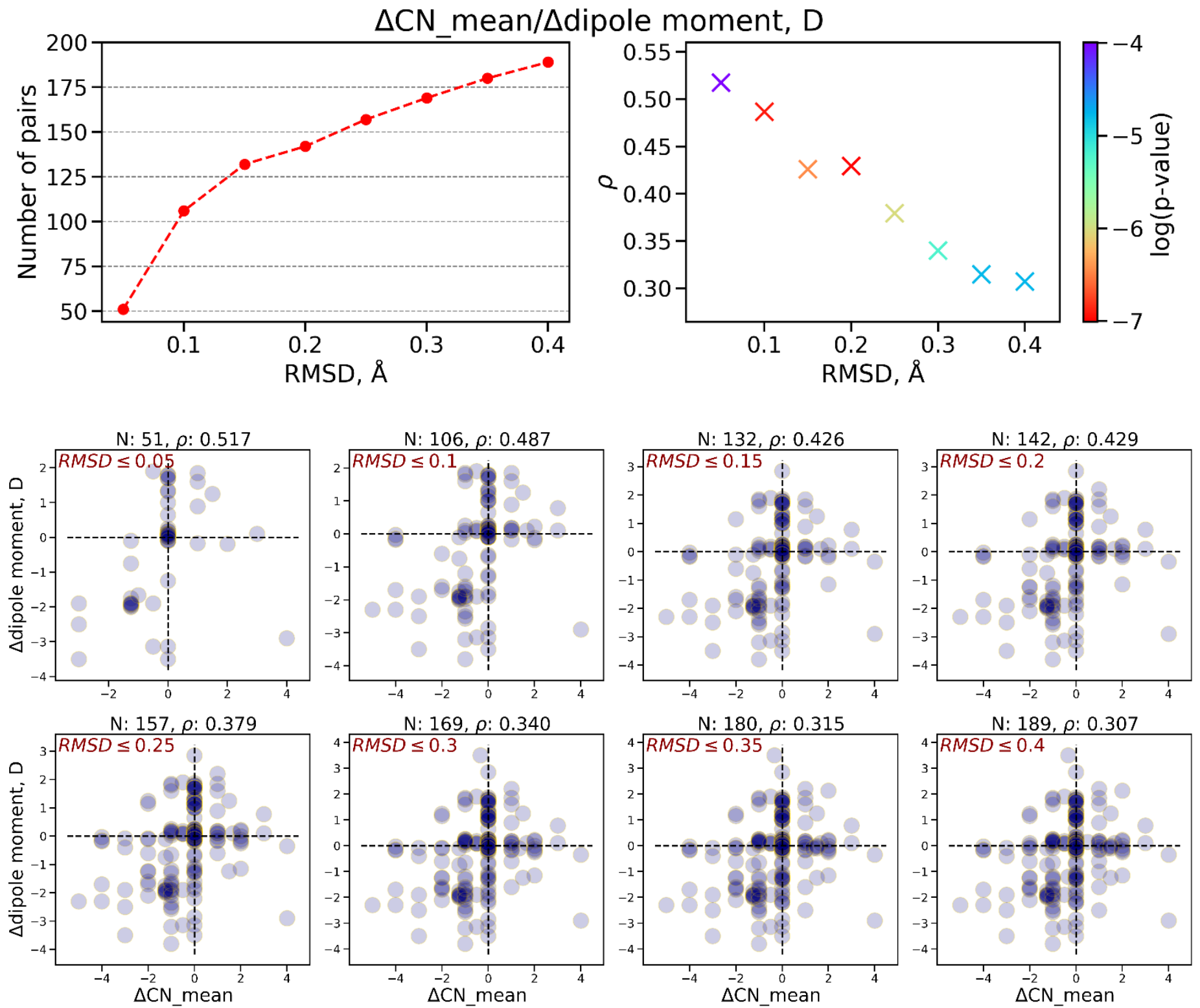
$\triangle \mathrm{CN}$ mean $/ \triangle \mathrm{nHBAcc}$
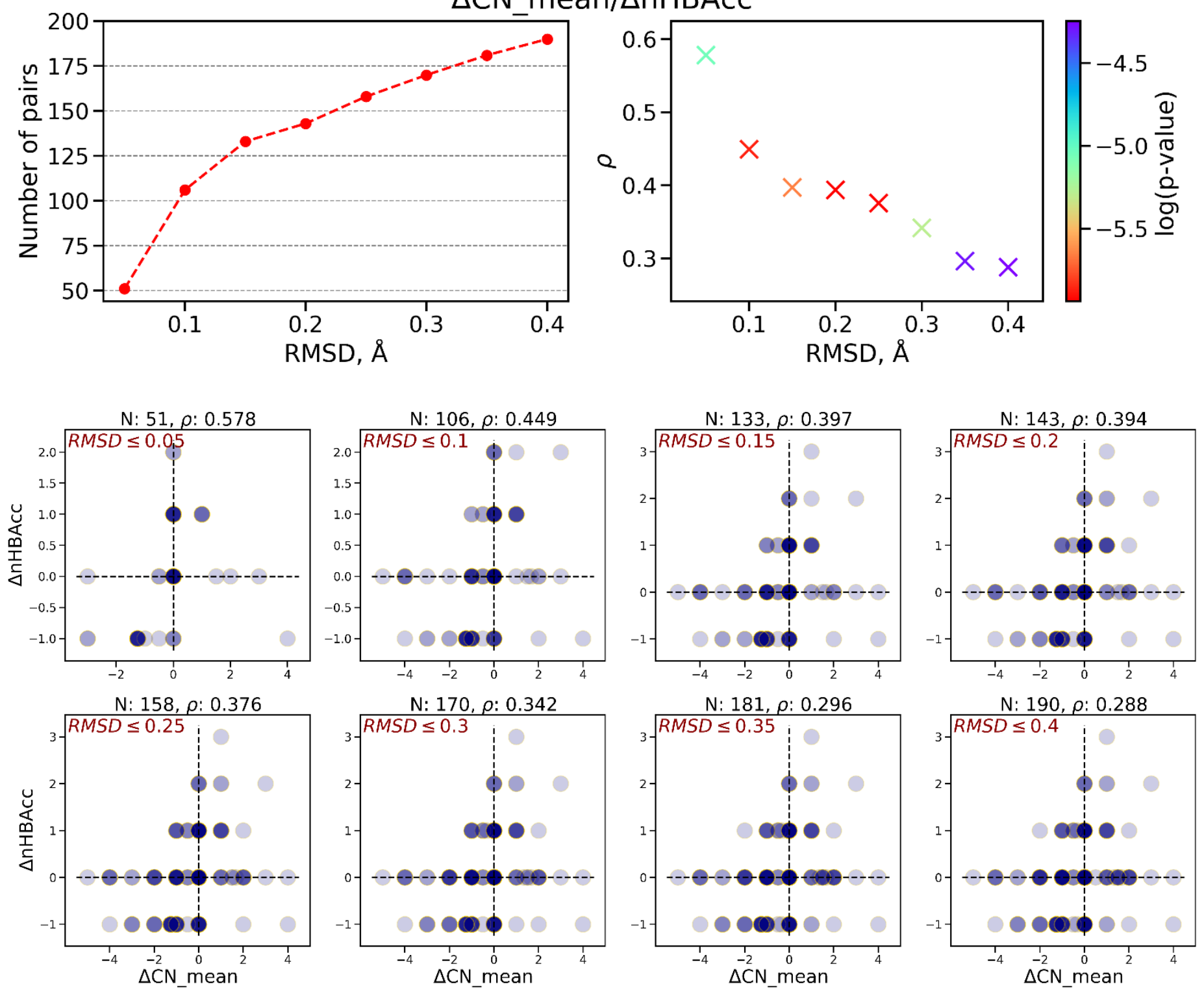

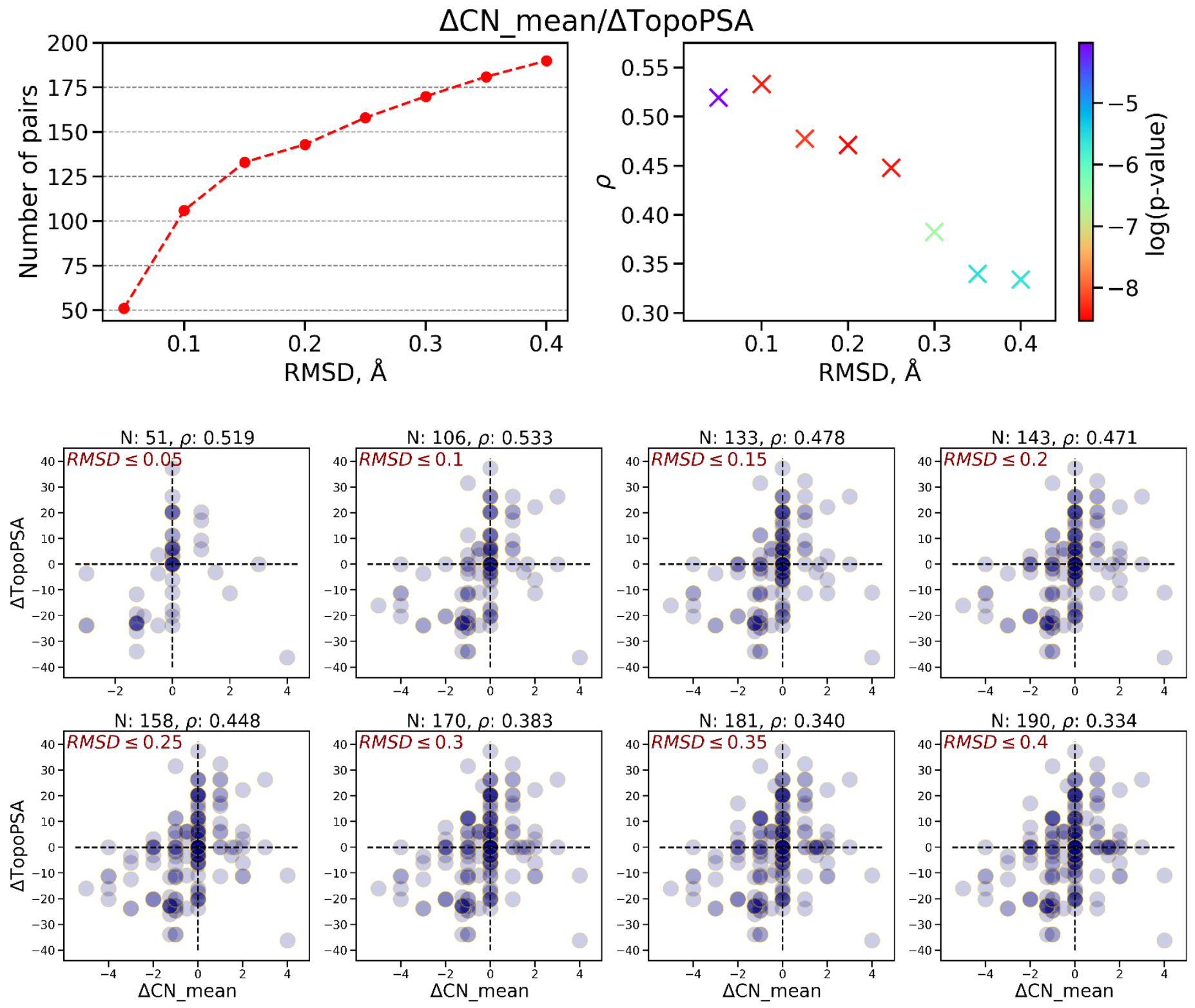

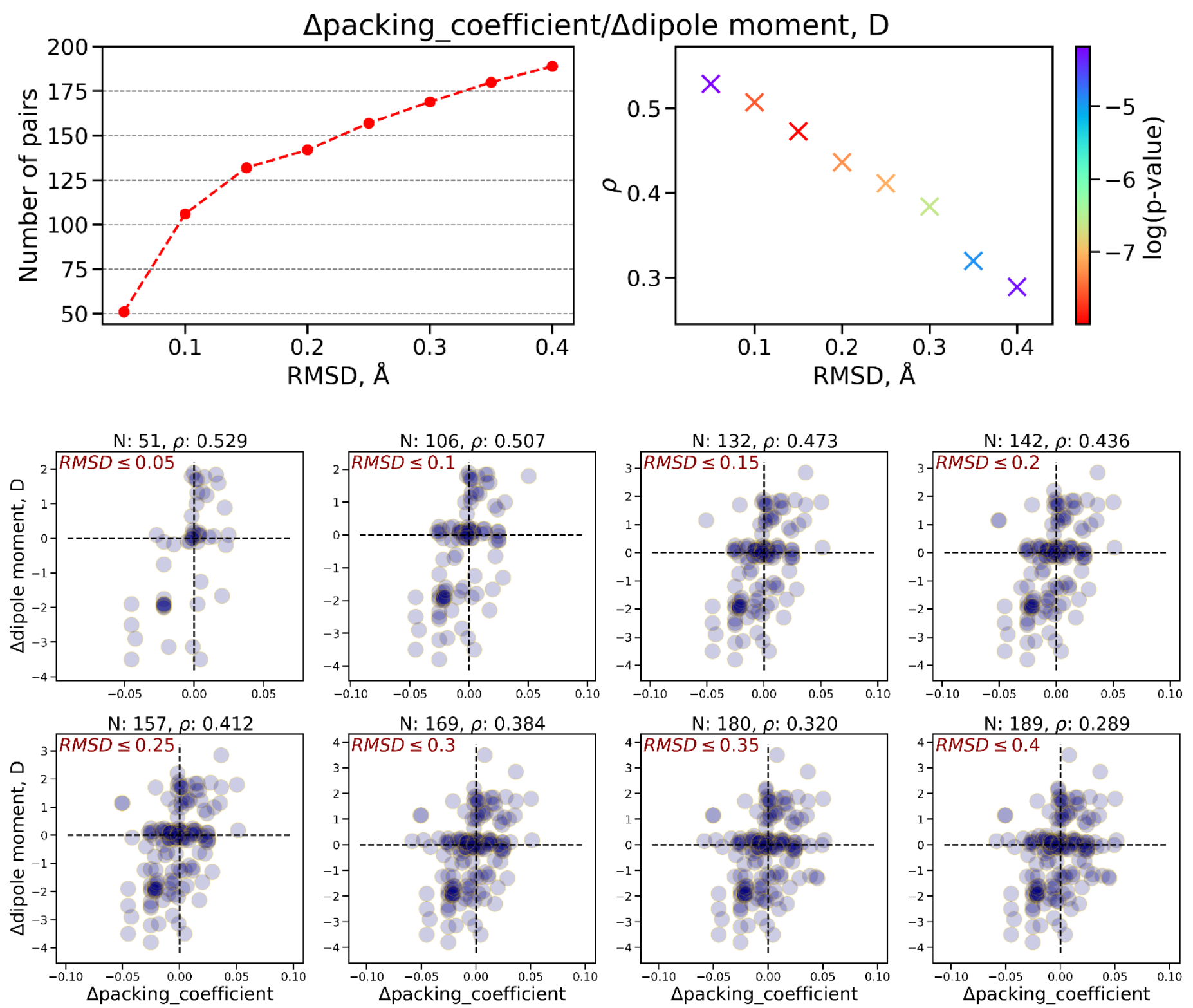

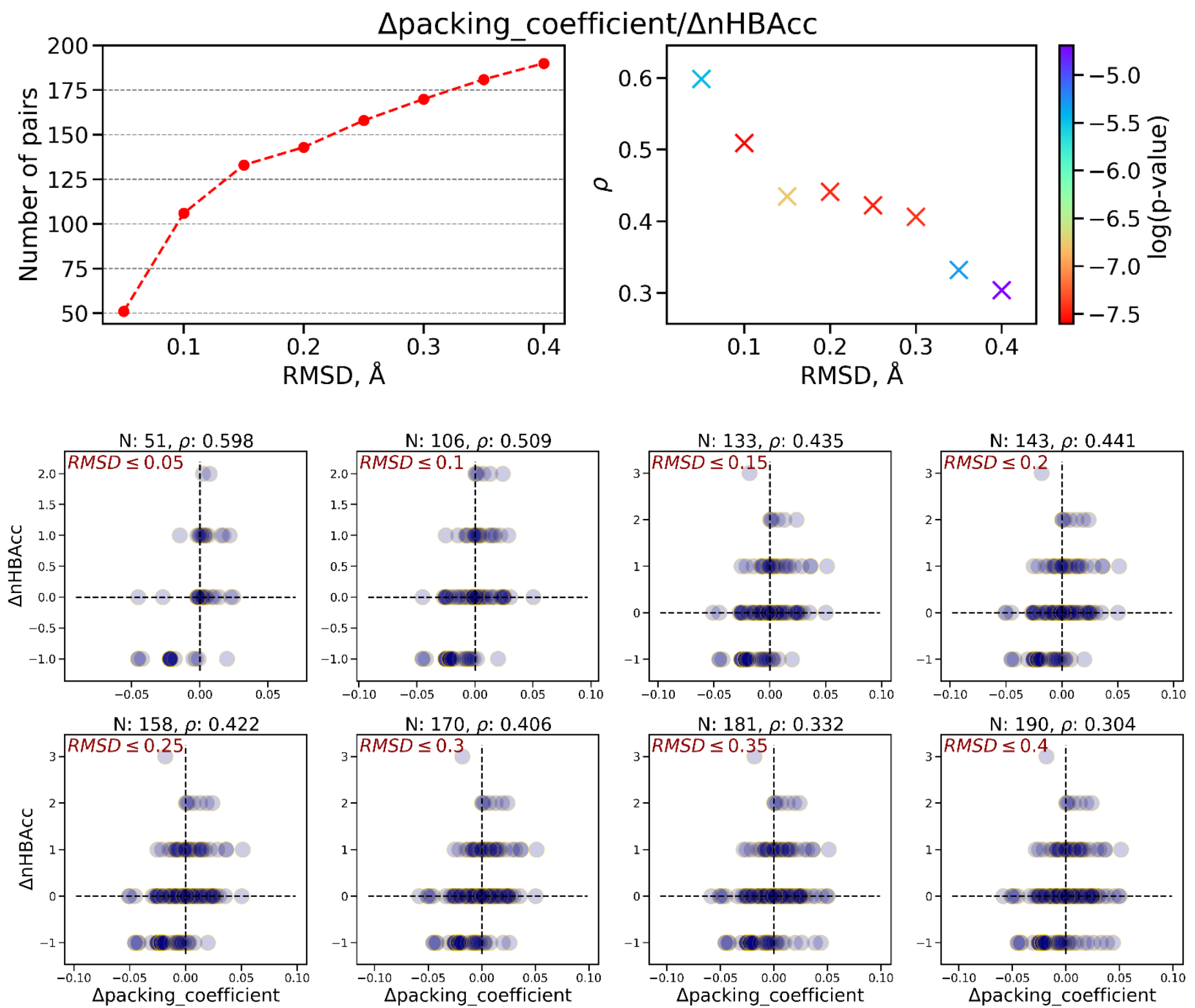

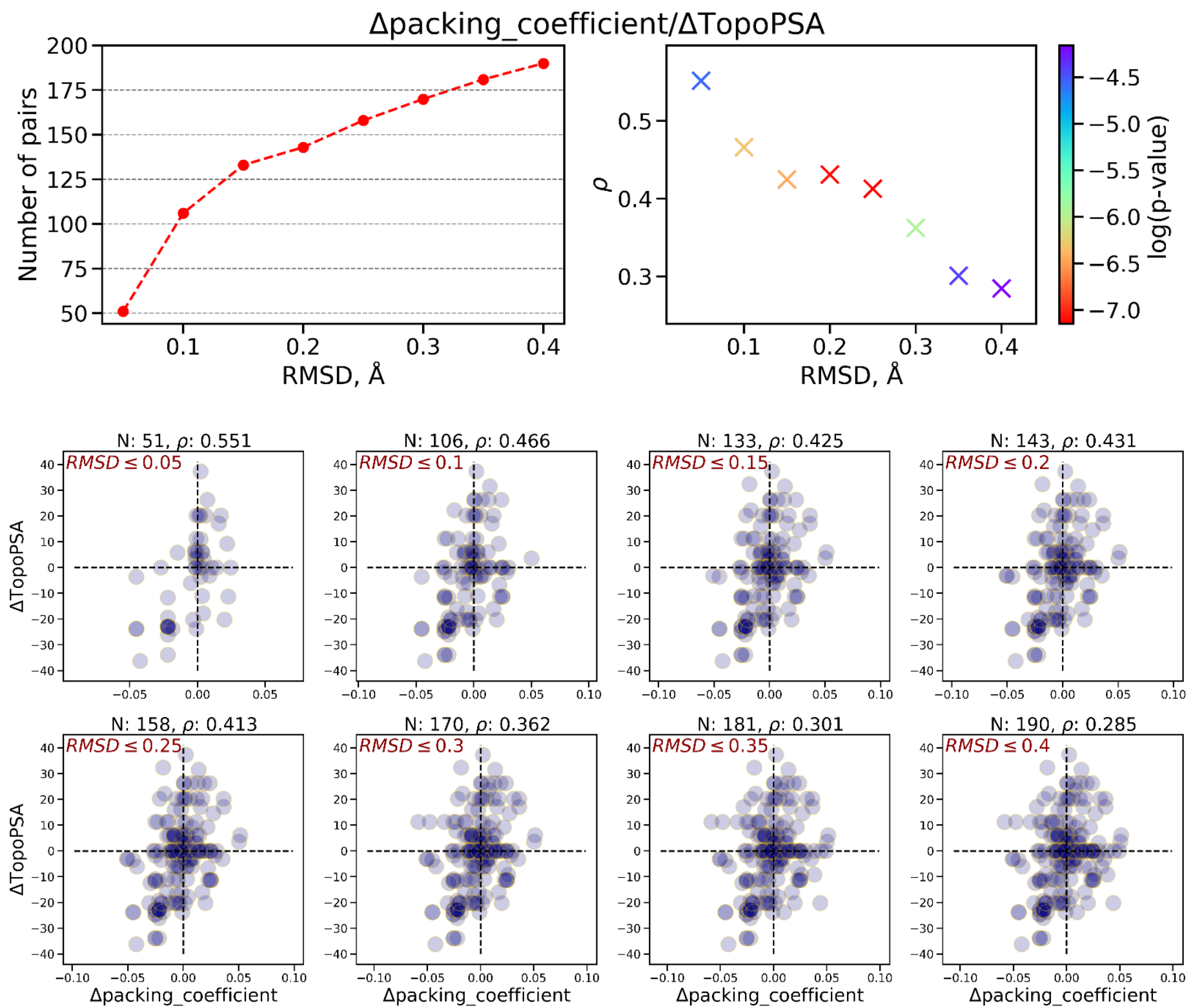

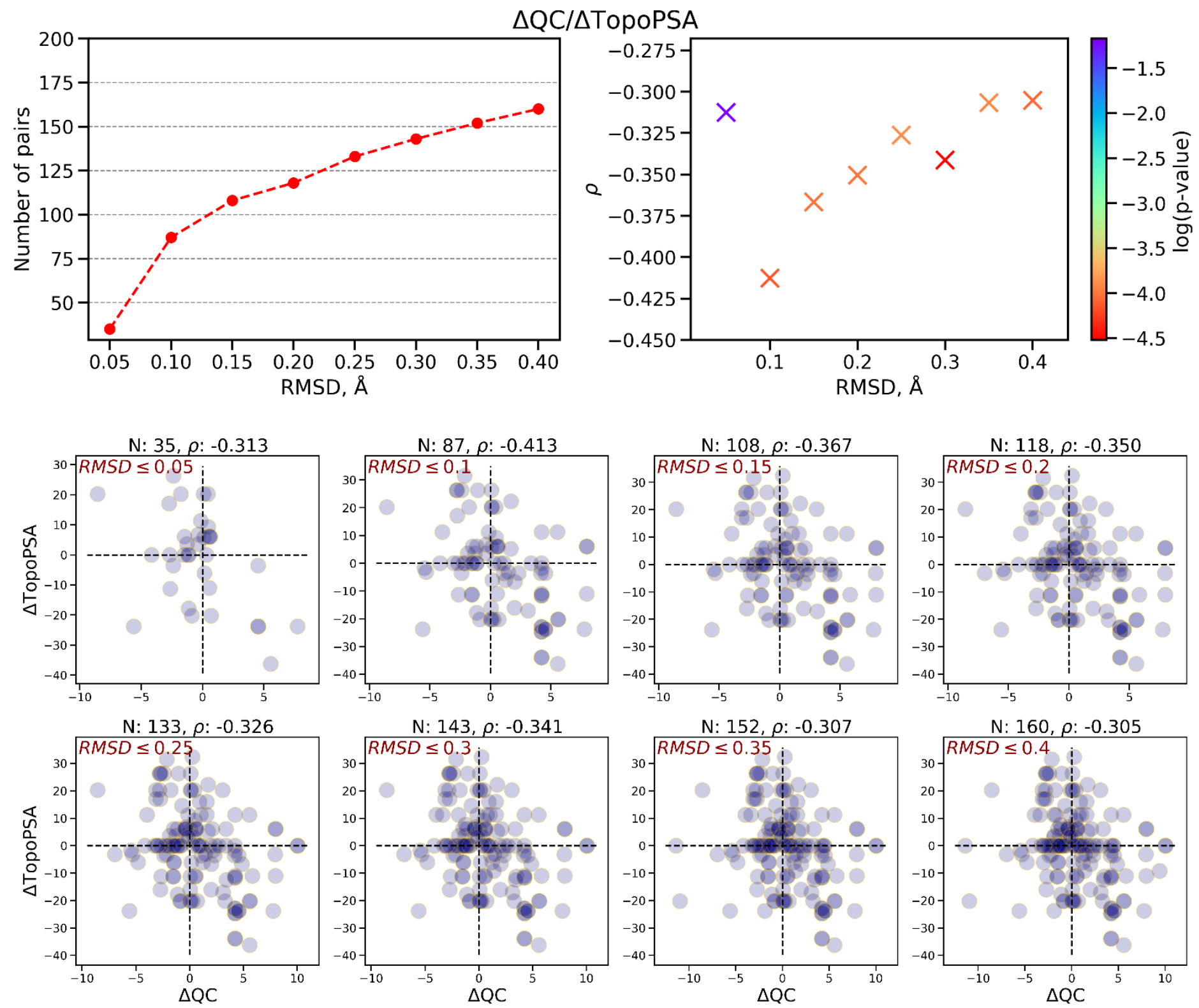

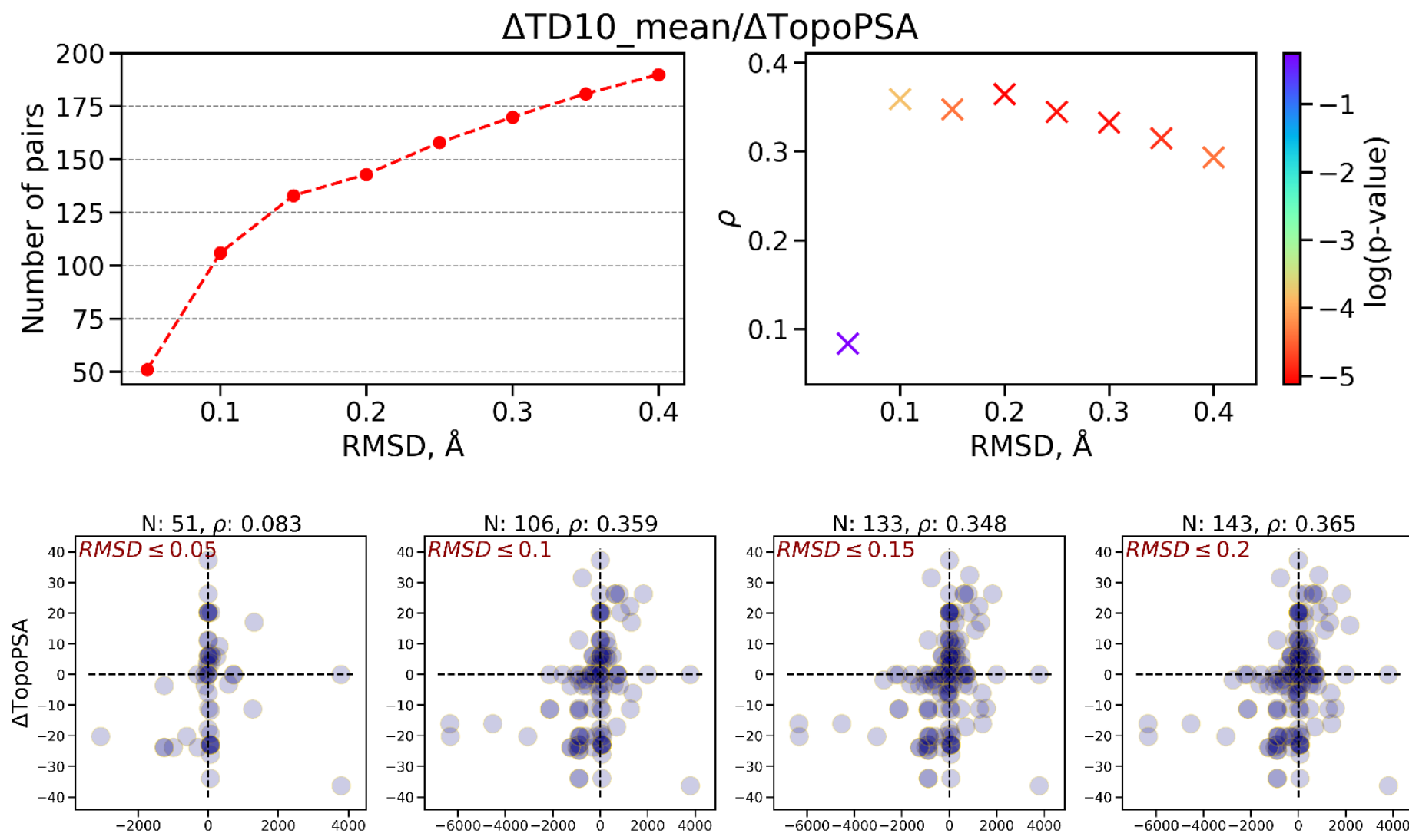

$\mathrm{N}: 106, \rho: 0.359$
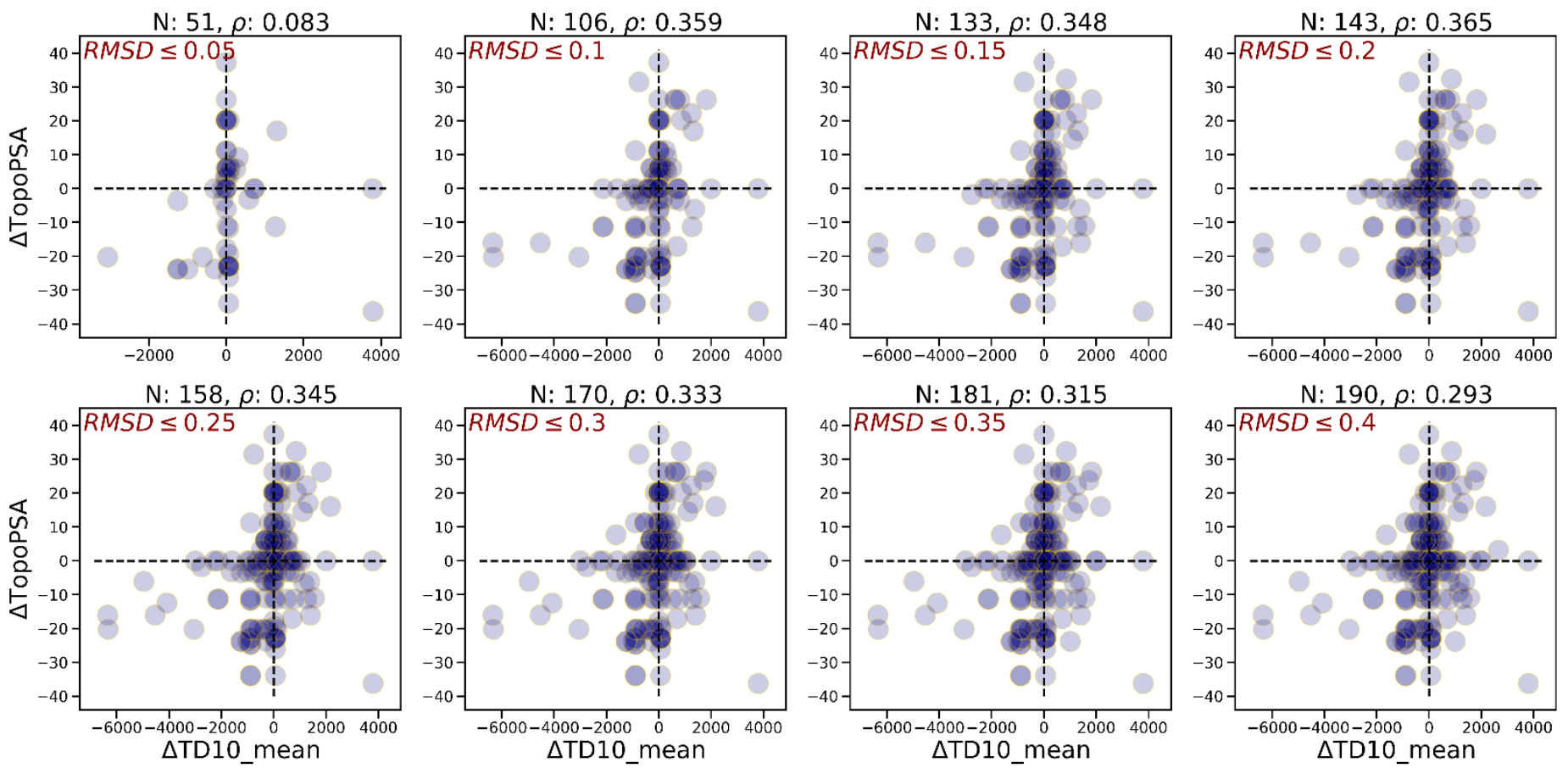
Fig. S5. The same scatterplots as in Fig. 3, but now separately for less polar $(\mu<3.615 \mathrm{D}$, dark blue) and more polar $(\mu>3.615 \mathrm{D}$, dark red) compounds as compared with the median molecular dipole moment 3.615 Debye (see the column "mean_dipole" in spreadsheet Table X10). Spearman's correlation coefficient values for two groups are shown in corresponding colour. Correlation coefficient for the whole set is shown in black. More pronounced response on solvent polarity change is seen for more polar compounds, as evaluated by Spearman's correlation coefficient values indicating closer to moderate correlation strength. Interactive versions of the mentioned scatterplots are available at https://trioxane.github.io/Fig_i5.html.
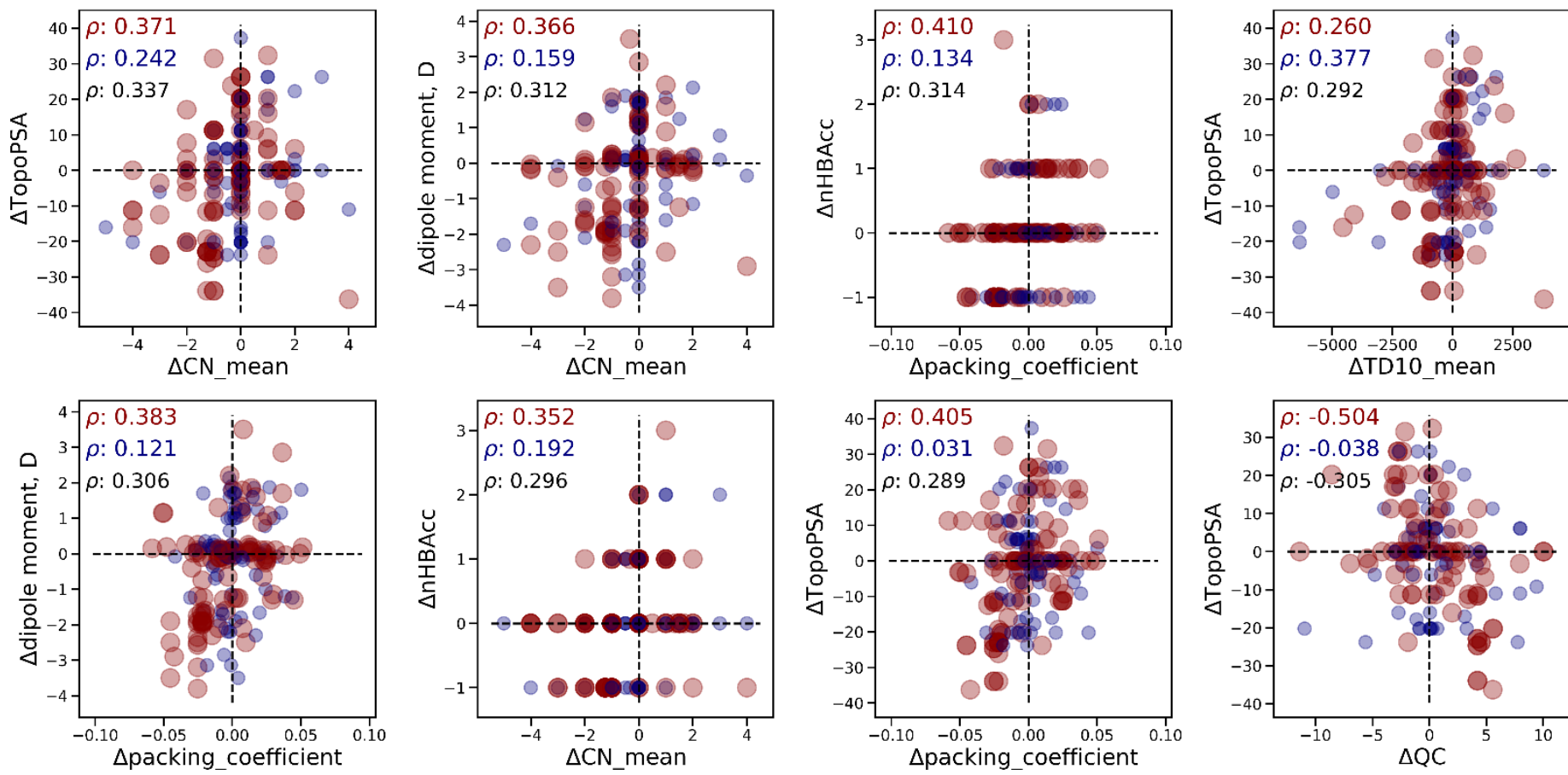

Fig. S6. Histograms of Pearson (left) and Spearman (right) correlation coefficients between 496 pairs of crystal molecule and solvent descriptor difference values.

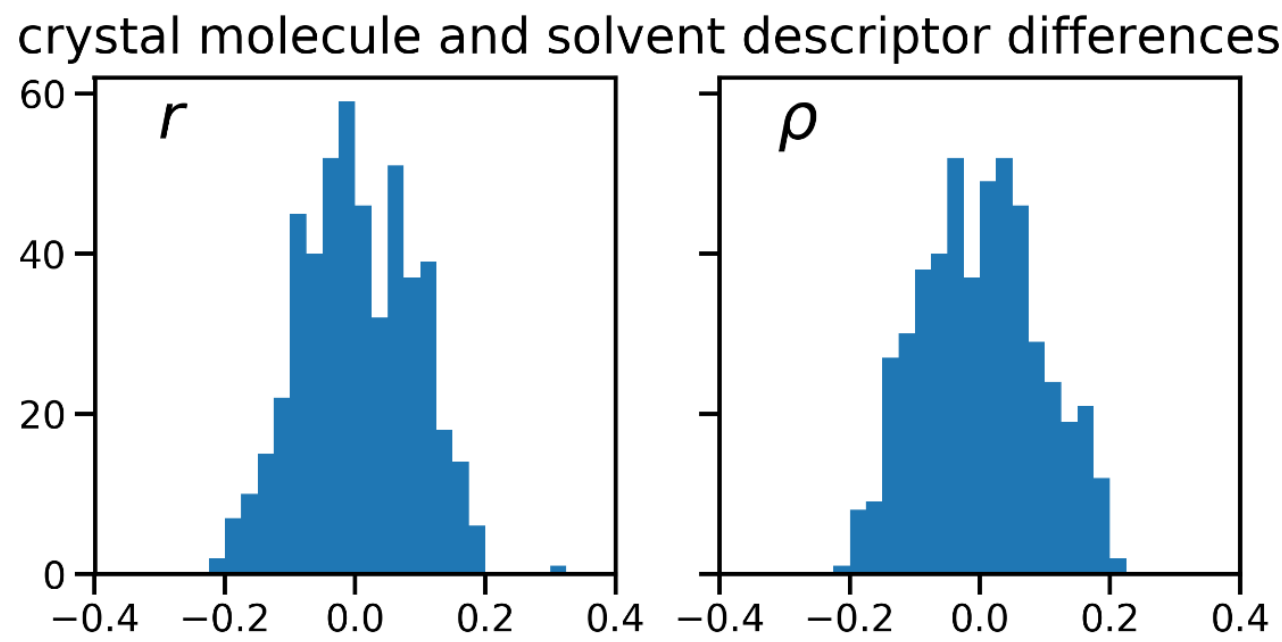

